Functional Analysis and Its Applications, Vol. 44, No. 1, pp. 4-21, 2010

Translated from Funktsional'nyi Analiz i Ego Prilozheniya, Vol. 44, No. 1, pp. 4-26, 2010

Original Russian Text Copyright (c) by F. V. Weinstein

\title{
Filtering Bases and Cohomology of Nilpotent Subalgebras of the Witt Algebra and the Algebra of Loops in $s l_{2}$
}

\section{F. V. Weinstein}

Received February 23, 2008

\begin{abstract}
We study the cohomology with trivial coefficients of the Lie algebras $L_{k}, k \geqslant 1$, of polynomial vector fields with zero $k$-jet on the circle and the cohomology of similar subalgebras $\mathscr{L}_{k}$ of the algebra of polynomial loops with values in $s l_{2}$. The main result is a construction of special bases in the exterior complexes of these algebras. Using this construction, we obtain the following results. We calculate the cohomology of $L_{k}$ and $\mathscr{L}_{k}$. We obtain formulas in terms of Schur polynomials for cycles representing the homology of these algebras. We introduce "stable" filtrations of the exterior complexes of $L_{k}$ and $\mathscr{L}_{k}$, thus generalizing Goncharova's notion of stable cycles for $L_{k}$, and give a polynomial description of these filtrations. We find the spectral resolutions of the Laplace operators for $L_{1}$ and $\mathscr{L}_{1}$.
\end{abstract}

Dedicated to the hallowed memory of my mother, Maria Weinstein

Let $h^{3}=1$, where $h \in \mathbb{C}$. Denote by $L^{(h)}$ the Lie algebra spanned over the field $\mathbb{Q}$ of rationals by finite linear combinations of basis vectors $e_{a}$, where $a$ runs over the ring $\mathbb{Z}$ of integers and the commutator is defined by the formula

$$
\left[e_{a}, e_{b}\right]=(b-a)_{h} e_{a+b}, \quad \text { where }(m)_{h}=\frac{h^{2 m}-h^{m}}{h^{2}-h}, m \in \mathbb{Z}
$$

The algebra $L^{(1)}$ is isomorphic to the algebra spanned by the derivations $e_{a}=t^{a+1} d / d t$ of the Laurent polynomial ring $\mathbb{Q}\left[t^{-1}, t\right]$. This Lie algebra is called the Witt algebra. The Lie algebra $L^{(h \neq 1)}$ is isomorphic to the loop algebra $s l_{2}(\mathbb{Q}) \otimes \mathbb{Q}\left[t^{-1}, t\right]$ on $s_{2}(\mathbb{Q})$ (see $[8$, Exercise 7.12$]$ ).

It seems to me that one cause for numerous analogies between these two algebras is the possibility to represent their commutators in the parametric form (1). For example, each of these algebras has a unique (up to an isomorphism) universal central extension, and the 2-cocycles determining these central extensions can be represented in the unified form

$$
\left(e_{i}, e_{j}\right) \rightarrow \delta_{i+j, 0} \kappa_{h}(i), \quad \text { where } \kappa_{h}(i)=\frac{1}{2} \sum_{r=1}^{i}(i-2 r+1)_{h}^{2}= \begin{cases}\left(\begin{array}{c}
i+1 \\
3
\end{array}\right) & \text { if } h=1 \\
\left\lfloor\frac{i+1}{3}\right\rfloor & \text { if } h \neq 1\end{cases}
$$

(Here $\lfloor a\rfloor$ is the integer part of a number a.) For $h=1$, this is the Gelfand-Fuchs cocycle, and the corresponding extension is called the Virasoro algebra. For $h \neq 1$, we obtain the affine algebra $A_{1}^{(1)}$.

Let $L^{(h)}(k) \subset L^{(h)}$ be the subalgebra generated by the vectors $e_{a}$ with $a \geqslant k \geqslant-1$. We sometimes write $L_{k}:=L^{(1)}(k)$ and $\mathscr{L}_{k}:=L^{(h \neq 1)}(k)$. For $k \geqslant 1$, these algebras are nilpotent. The main result of this paper is a construction of special bases in the exterior complexes of the algebras $L^{(h)}(k)$ for $k \geqslant 1$. One application of this construction is the computation of the continuous cohomology spaces $H^{*}\left(L^{(h)}(k) ; \mathbb{Q}\right)$, where $\mathbb{Q}$ is treated as a trivial $L^{(h)}(k)$-module and continuous $\mathbb{Q}$-linear functionals on $L^{(h)}(k)$ are those with finite-dimensional supports. In particular, we prove that

$$
\sum_{q=0}^{\infty} \operatorname{dim} H^{q}\left(L^{(h)}(k) ; \mathbb{Q}\right) t^{q}=\frac{1+t}{(1-t)^{k}} .
$$


For $h=1$, this formula (in a different form; see Remark 2.6) was included in Gelfand's talk [5] as a conjecture.* The first published proof of the conjecture by Goncharova [7] is very cumbersome. Later, several proofs of her theorem on the basis of different, very interesting ideas were found for $k=1$ (e.g., see [2] and [11]). ${ }^{* *}$

Our work is motivated by two sources. The first one is Goncharova's paper [7], where she introduced the definitions, important to us, of non-singular and main k-partitions and stable cycles in the exterior complexes of the algebras $L_{k}$. Goncharova's main result is a proof of the existence of a stable cycle for each main $k$-partition and of the fact that the homological classes of these cycles form a basis of the homology space $H_{*}\left(L_{k} ; \mathbb{C}\right)$.

The second source is the paper [6] by Gelfand, Feigin, and Fuchs. On the exterior complex of the algebra $L_{1}$, they consider the Euclidean structure in which the vectors $e_{i_{1}} \wedge \cdots \wedge e_{i_{q}}$ are orthonormal. Then there is a linear self-adjoint "Laplace operator" $\Gamma$ naturally defined on the complex. The kernel of $\Gamma$ is isomorphic to the homology of $L_{1}$. The main, quite unexpected observation in [6] is that the eigenvalues of this operator are explicitly computable integers. Additionally, a nice description of the stable cycles of $L_{k}$ is given.

Unfortunately, the paper [6] is not completely correct. Namely, the main tool of analysis in [6] - the construction proposed there for the eigenvectors of $\Gamma$-often fails. As a consequence, the statement of the main result contains an error: the claimed multiplicities of the eigenvalues of $\Gamma$ are wrong, although the eigenvalues themselves are correct.

The desire to clarify the ideas and results in [6] and [7] and understand the relations between them has been the main motivation for me. The paper is organized as follows.

Section 1 gathers some material pertaining to partitions and used throughout the paper. The main result is stated in Section 2. Namely, in terms of the marked $k$-partitions introduced in Section 1 , we define $\varepsilon(k)$-monomials as certain vectors in the exterior complex $C_{*}(k)$ of $L^{(h)}(k)$. The main Theorem 2.4 claims that these vectors form a basis of the complex and that this basis has some "filtering" properties. Then, taking this theorem for granted, we compute the cohomology space $H^{*}\left(L^{(h)}(k) ; \mathbb{Q}\right)$.

The proof of the main theorem is contained in Section 3. First, we establish some identities, playing a key role in what follows, in the exterior complexes of $L^{(h)}(k)$ (Lemma 3.1). We use these identities to prove that the $\varepsilon(1)$-monomials generate $C_{*}(1)$ and have the properties claimed in Theorem 2.4. The linear independence of the $\varepsilon(1)$-monomials follows from a formula that expresses the number of integer partitions of positive integers with pairwise distinct parts via such monomials. This formula proves to be equivalent to Sylvester's classical identity

$$
\prod_{i=1}^{\infty}\left(1+t x^{i}\right)=1+t x \frac{1+t x^{2}}{1-x}+\sum_{l=2}^{\infty} t^{l} x^{\left(3 l^{2}-l\right) / 2} \frac{(1+t x) \cdots\left(1+t x^{l-1}\right)\left(1+t x^{2 l}\right)}{(1-x) \cdots\left(1-x^{l-1}\right)\left(1-x^{l}\right)}
$$

in partition theory (see [12, p. 282]). The proof of the theorem is completed by induction on $k$ with the use of the above-mentioned identities and an additional construction for marked partitions.

It seems to be quite interesting that Sylvester's identity arises when constructing the filtering basis. But it is somewhat disappointing that we rely on this identity in the proof of the main theorem. Therefore, in the additional Section 8 I give a bijective proof of the desired combinatorial fact that is shown in Section 3 to be equivalent to this identity. This equivalence provides a new interpretation of Sylvester's identity.

The subsequent sections contain applications of the main theorem. In Section 4, we introduce two filtrations of the complex $C_{*}(k)$, an increasing "stable" filtration whose initial term is the space of stable cycles and a decreasing $\varepsilon$-filtration. The definition of the stable filtration uses only the definition of the complex $C_{*}(k)$, while to define the $\varepsilon$-filtration we need the main theorem. In Section 4 , we show that these filtrations are dual with respect to the Euclidian structure considered.

*As far as I know, this conjecture is due to D. B. Fuchs.

${ }^{* *}$ In [2], the cases of $k=2,3$ are considered as well, but the treatment is ad hoc and cannot be generalized to $k>3$. 
In Section 5, we follow an idea in [6] and identify the chains of algebras $L^{(h)}(k)$ with skewsymmetric polynomials. Using this identification, we obtain a formula for the action of the boundary operator in the exterior complex. This formula generalizes the corresponding formula in [6] to $L^{(h)}(k)$. The approach to stable cycles suggested in [6] is generalized as well and gives a polynomial description of the stable filtration.

A comparison of the results in Sections 4 and 5 leads to a family of bases in the symmetric polynomial rings with finitely many variables (Corollary 5.4). Such a basis depends on the parameters $h$ and $k$, consists of homogeneous polynomials, and is numbered by marked $k$-partitions. For $k=1$, this gives a combinatorial formula for the dimension of the space of homogeneous polynomials $f\left(t_{1}, \ldots, t_{q}\right)$ of fixed degree over $\mathbb{Q}$ with the property

$$
f\left(h t_{1}, \bar{h} t_{1}, \ldots, h t_{m}, \bar{h} t_{m}, t_{m+1}, \ldots, t_{q-m}\right)=0,
$$

where $0 \leqslant m \leqslant\lfloor q / 2\rfloor, h^{3}=1$, and $\bar{h}$ is the complex conjugate of $h$. This dimension is independent of the choice of $h$.

In Section 6, the results obtained are used for computing the homology of $L^{(h)}(k)$. First, we build a basis of $H_{*}\left(L^{(h)}(k) ; \mathbb{Q}\right)$ represented by stable cycles, which extends Goncharova's result to the algebras $L^{(h)}(k)$. Next, we obtain explicit formulas for these cycles via Schur polynomials.

In Section 7, we consider the Laplace operator $\Gamma^{(h)}$ for the algebra $L^{(h)}(1)$. We prove that the action of $\Gamma^{(h)}$ is expressed in the basis of $\varepsilon(1)$-monomials by a rational triangular matrix whose diagonal entries can be computed explicitly. In particular, this gives the spectrum of $\Gamma^{(h)}$. Using this, we show that the multiplicities of the eigenvalues of $\Gamma^{(1)}$ are finite and their computation can be reduced to a number-theoretic problem. In the basis of $\varepsilon(1)$-monomials, the action of $\Gamma^{(h \neq 1)}$ is diagonal, the spectrum of $\Gamma^{(h \neq 1)}$ is the set $\mathbb{Z}_{\geqslant 0}$, and the multiplicity of each eigenvalue is infinite. One corollary of our calculations is the invariance of the stable filtration with respect to the action of $\Gamma^{(h)}$. This generalizes the claim in [6] concerning the action of the Laplacian on the space of stable cycles of the algebra $L_{1}$. For $h \neq 1$, our result on the spectral resolution of $\Gamma^{(h)}$ is a well known special case of a much more general fact (see [9, 3.2-3.4]).

Many interesting questions about the cohomology of the algebras $L^{(h)}(k)$ can be reduced to questions about the expansions of their cochains in the filtering basis. The problem of computing the multiplicative structure of the rings $H^{*}\left(L^{(h)}(k) ; \mathbb{Q}\right.$ ) (see Remark 2.7) can serve as an example. The problem of describing the structure of $H^{*}\left(L^{(h)}(k) ; \mathbb{Q}\right)$ as an $L^{(h)}(1) / L^{(h)}(k)$-module for $k \geqslant 2$ (see [1] and [3]) is another example. In full generality, these problems are open yet.

Part of the results presented here were already published in [13] and [14], ${ }^{*}$ where the algebras $L_{k}$ of vector fields were considered. In this paper, in addition to technical simplifications and new results, we simultaneously consider the algebras $L^{(h)}(k)$ for $h=1$ and $h \neq 1$ in the framework of a unified approach and emphasize the analogy between them.

Notation. The symbols $\alpha, \beta$, and $\gamma$ (with or without subscripts) denote rational numbers. Unless otherwise specified, all vector spaces are assumed to be defined over $\mathbb{Q} ; k$ stands for a positive integer. The cardinality of a set $M$ is denoted by $|M|$. For $x, y \in M$, the function $\delta_{x, y}$ is the Kronecker delta. The exterior algebra of a vector space $V$ is denoted by $\Lambda(V)=\bigoplus_{q=0}^{\infty} \Lambda^{q}(V)$, where $\Lambda^{q}(V)$ is the $q$ th exterior power of $V$. To refer to the algebras $L^{(h)}(k)$ with different $h$ simultaneously, we write $L(k)$. For various objects related to these algebras, we usually omit $h$ and specify which $h$ is meant where necessary.

\section{Nonsingular, Main, and Marked Partitions}

This section introduces the main definitions and notation concerning partitions and marked partitions, to be used throughout the paper.

${ }^{*}$ The paper [13] contains a mistake, corrected in [14], in the definition of the spectral sequence. 
Definition 1.1. A partition is a finite ordered set $I=\left[i_{1}, \ldots, i_{q}\right]$ of integers, referred as the parts of the partition, such that $0 \leqslant i_{1} \leqslant \cdots \leqslant i_{q}$. A partition is said to be strict if $i_{1}<\cdots<i_{q}$. We write $I^{-}=i_{1}$ and $I^{+}=i_{q}$.

The numbers $\|I\|=i_{1}+\cdots+i_{q}$ and $|I|=q$ are called the degree and length of $I$, respectively.

A subpartition of $I$ is an ordered subset $I^{\prime} \subset I$. The union $I_{1} \sqcup I_{2}$ of partitions $I_{1}$ and $I_{2}$ is the partition whose set of parts is the disjoint union of the sets of parts of $I_{1}$ and $I_{2}$.

Definition 1.2. A marked partition is an equivalence class of the pairs $[I ; J]$, where $I$ is a partition, $J \subset I$, and $[I ; J]$ is equivalent to $\left[I_{1} ; J_{1}\right]$ if $I=I_{1}$ and $J=J_{1}$ as partitions. The elements of $J$ are called the marked parts. We identify each partition $I$ with the marked partition $[I ; \varnothing]$. The numbers $\|I\|$ and $|I|$ are called the degree and length of $[I ; J]$, respectively. We set $\left[I_{1} ; J_{1}\right] \sqcup\left[I_{2} ; J_{2}\right]=\left[I_{1} \sqcup I_{2} ; J_{1} \sqcup J_{2}\right]$. The set of marked partitions is denoted by $\mathbf{D}$.

When this cannot lead to a confusion, we refer to marked partitions simply as partitions. Instead of explicitly indicating the set of marked parts, we often underline these parts, $[[1,4,6,7] ;[4,7]]=$ $[1, \underline{4}, 6, \underline{7}]$. Note that, by definition, one has, e.g., $[\underline{5}, 5]=[5, \underline{5}]$.

Definition 1.3. Let $I$ and $I^{\prime}$ be distinct partitions. We write $I^{\prime} \triangleleft I$ ( $I^{\prime}$ is less than $I$ ) if $\left|I^{\prime}\right|=|I|,\left\|I^{\prime}\right\|=\|I\|$, and

$$
i_{1}^{\prime}+\cdots+i_{r}^{\prime} \leqslant i_{1}+\cdots+i_{r} \text { for all } r, 1 \leqslant r \leqslant|I| .
$$

Definition 1.4. Let $[I ; J]$ and $\left[I^{\prime} ; J^{\prime}\right]$ be distinct marked partitions. We write $\left[I^{\prime} ; J^{\prime}\right] \triangleleft[I ; J]$ if $\left|I^{\prime}\right|+\left|J^{\prime}\right|=|I|+|J|$ and either $\left|I^{\prime}\right|<|I|$, or $I^{\prime} \triangleleft I$, or $I^{\prime}=I$ and $J^{\prime} \prec J$, where $\prec$ stands for the lexicographic order.

For example, $[\underline{5}] \triangleleft[2,3],[\underline{3}, 6] \triangleleft[3, \underline{6}]$, and $[\underline{3}, 6,10, \underline{12}] \triangleleft[3, \underline{6}, \underline{10}, 12]$.

One can readily show that $₫$ is a partial order on $\mathbf{D}$.

Claim $(1.16)^{*}$ in $[10]$ can be restated as follows:

Lemma 1.5. For integer $a$ and $b, 1 \leqslant a<b \leqslant q$, let $R_{a, b}: \mathbb{Z}^{q} \rightarrow \mathbb{Z}^{q}$ be the mapping defined by the formula

$$
R_{a, b}\left(i_{1}, \ldots, i_{q}\right)=\left(i_{1}, \ldots, i_{a}+1, \ldots, i_{b}-1, \ldots, i_{q}\right) .
$$

If $I^{\prime} \triangleleft I$ and $\left|I^{\prime}\right|=|I|$, then there exists a sequence of pairs $\left(a_{r}, b_{r}\right)$ of integers, $1 \leqslant a_{r}<b_{r} \leqslant q$, such that $I^{\prime}=I_{1} \triangleleft \cdots \triangleleft I_{m}=I$, where $I_{r+1}=R_{a_{r}, b_{r}}\left(I_{r}\right)$.

Lemma 1.6. If $I^{\prime} \triangleleft I$ and $I_{1}^{\prime} \triangleleft I_{1}$, then $I^{\prime} \sqcup I_{1}^{\prime} \triangleleft I \sqcup I_{1}$.

Proof. It suffices to consider the case in which $I=I^{\prime}$. By Lemma 1.5, it suffices to show that $I \sqcup R_{a, b}\left(I_{1}^{\prime}\right) \triangleleft I \sqcup I_{1}^{\prime}$ for $a$ and $b$ such that $R_{a, b}\left(I_{1}^{\prime}\right) \triangleleft I_{1}^{\prime}$. But this is obvious.

Corollary 1.7. If $\left[I^{\prime} ; J^{\prime}\right] \preccurlyeq[I ; J]$ and $\left[I_{1}^{\prime} ; J_{1}^{\prime}\right] \triangleleft\left[I_{1} ; J_{1}\right]$, then $\left[I^{\prime} ; J^{\prime}\right] \sqcup\left[I_{1}^{\prime} ; J_{1}^{\prime}\right] \triangleleft[I ; J] \sqcup\left[I_{1} ; J_{1}\right]$.

Definition $1.8[7]$. A partition $\left[i_{1}, \ldots, i_{q}\right]$ is said to be nonsingular if $i_{r+1}-i_{r} \geqslant 3,1 \leqslant r \leqslant$ $q-1$. A dense partition is a partition of the form $\xi(a, q)=[a, a+3, \ldots, a+3(q-1)]$.

Definition 1.9. A $k$-partition is a pair $\{k ; I\}$, where $I$ is a partition and $I^{-} \geqslant k$. A $k$-partition is said to be nonsingular if $I$ is a nonsingular partition.

We write $k$-partitions as usual partitions, emphasizing that we only consider $k$-partitions. For example, one may treat $[2,6]$ as either a 1-partition or a 2-partition. These objects are not the same.

Definition 1.10 [7]. A main $k$-partition is a nonsingular $k$-partition $\left[i_{1}, \ldots, i_{q}\right]$ such that

$$
i_{q} \leqslant 2 k+3(q-1) \quad \text { for } i_{1}>k \text { and } i_{q}<2 k+3(q-1) \text { for } i_{1}=k .
$$

For example, $\xi(1, q)$ and $\xi(2, q)$ exhaust all main 1-partitions of length $q \geqslant 1$.

Definition 1.11. For a nonsingular $k$-partition $I$, there exists a unique decomposition $I=$ $I_{1} \sqcup \cdots \sqcup I_{s}$, called the standard form of $I$, where $I_{1}, \ldots, I_{s}$ are nonsingular $k$-partitions such that $I_{a+1}^{-}-I_{a}^{+}>3,1 \leqslant a \leqslant s-1$, and the following two conditions are satisfied:

(1) If $I^{-} \leqslant 2 k$, then $I_{1}$ is a main subpartition of $I$ of maximum possible length. It is called the main component of $I$. 
(2) The partitions $I_{a}$ different from the main one are dense. They are called the dense components of $I$.

The parts $I_{r}^{-}$of the dense components $I_{r}$ are called the leading parts of $I$. The number of leading parts is called the index of $I$ and is denoted by $\operatorname{ind}_{k} I$.

For example, the standard form of $I=[2,5,9,14,17]$ is $[2,5] \sqcup[9] \sqcup[14,17]$ if $I$ is treated as a 1-partition and $[2,5,9] \sqcup[14,17]$ if $I$ is treated as a 2-partition. Hence $\operatorname{ind}_{1} I=2$ and $\operatorname{ind}_{2} I=1$.

Definition 1.12. A marked $k$-partition is a pair $\{k ;[I ; J]\}$, where $[I ; J] \in \mathbf{D}, I$ is a $k$-partition, and the partition $I \backslash J$ is strict.

Definition 1.13. A marked $k$-partition $[I ; J]$ is said to be nonsingular if $I$ is a nonsingular $k$-partition and $J$ is a subset of the set of leading parts of $I$. Otherwise, $[I ; J]$ is said to be singular.

We use the following notation:

$\mathrm{N}(k)$ is the set of nonsingular $k$-partitions.

$\mathbf{D}(k)$ is the set of marked $k$-partitions.

$\mathbf{N}(k)$ is the set of nonsingular marked $k$-partitions.

$\mathrm{M}(k)$ is the set of main $k$-partitions.

$\mathrm{M}_{q}(k)$ is the set of main $k$-partitions of length $q$.

As the subsets of the set $\mathbf{D}$, all these sets inherit the partial order $\sharp$.

\section{Filtering Basis Theorem and the Cohomology of the Algebras $L(k)$}

Let $\left\{C_{*}(k), d\right\}$ be the exterior complex of the Lie algebra $L(k)$, where $C_{*}(k)=\bigoplus_{q \geqslant 0} C_{q}(k)$. Its space $C_{q}(k)=\Lambda^{q}(L(k))$ of $q$-dimensional chains consists of finite linear combinations of the basic vectors $e_{I}=e_{i_{1}} \wedge \cdots \wedge e_{i_{q}}$, called $k$-monomials, where $I=\left[i_{1}, \ldots, i_{q}\right]$ is a strict $k$-partition. The action of the boundary operator $d$ is defined as

$$
d_{k}\left(e_{I}\right)=\sum_{1 \leqslant s<t \leqslant q}(-1)^{s+t-1}\left(i_{t}-i_{s}\right)_{h} e_{i_{s}+i_{t}} \wedge e_{i_{1}} \wedge \cdots \wedge \widehat{e}_{i_{s}} \wedge \cdots \wedge \widehat{e}_{i_{t}} \wedge \cdots \wedge e_{i_{q}}
$$

Let $C_{*}^{(n)}(k)$ be the space spanned by the $k$-monomials $e_{I}$ with $\|I\|=n$. Then $C_{*}(k)=$ $\bigoplus_{n \geqslant 0} C_{*}^{(n)}(k)$ (a direct sum of complexes). We define the space of $q$-dimensional cochains as $C^{q}(k)=\bigoplus_{n \geqslant 0} C_{(n)}^{q}(k)$, where $C_{(n)}^{q}(k)=\operatorname{Hom}_{\mathbb{Q}}\left(C_{q}^{(n)}(k), \mathbb{Q}\right)$.

Let us introduce an inner product in $C_{*}(k)$ by setting $\left\langle e_{I_{1}}, e_{I_{2}}\right\rangle=\delta_{I_{1}, I_{2}}$. It defines isomorphisms $C_{q}^{(n)}(k) \cong C_{(n)}^{q}(k)$, which allow us to treat the chains of the algebra $L(k)$ as cochains. Let $\delta_{k}$ be the (coboundary) operator on $C_{*}(k)=C^{*}(k)$ dual to $d_{k}$ with respect to the inner product. The definitions imply the formulas

$$
\begin{gathered}
\delta_{k}\left(e_{i}\right)=\sum_{a+b=i ; k \leqslant a<b}(b-a)_{h} e_{a} \wedge e_{b}, \\
\delta_{k}\left(e_{i_{1}} \wedge \cdots \wedge e_{i_{q}}\right)=\sum_{1 \leqslant a \leqslant q}(-1)^{a-1} e_{i_{1}} \wedge \cdots \wedge \delta_{k}\left(e_{i_{a}}\right) \wedge \cdots \wedge e_{i_{q}} .
\end{gathered}
$$

The homology of the complexes $\left\{C_{*}(k), d_{k}\right\}$ and $\left\{C^{*}(k), \delta_{k}\right\}$ is called the homology and cohomology, respectively, of the algebra $L(k)$ and is denoted by $H_{*}(k)$ and $H^{*}(k)$.

Definition 2.1. Given $[I ; J] \in \mathbf{D}(k), I=\left[i_{1}, \ldots, i_{q}\right]$, define $e_{[I ; J]}=e_{\left(i_{1}\right)} \wedge \cdots \wedge e_{\left(i_{q}\right)}$, where

$$
e_{\left(i_{a}\right)}= \begin{cases}e_{i_{a}} & \text { if } i_{a} \notin J, \\ \delta_{k}\left(e_{i_{a}}\right) ; & \text { if } i_{a} \in J .\end{cases}
$$

A cochain of the form $e_{[I ; J]}$ is called an $e(k)$-monomial. It is called an $\varepsilon(k)$-monomial if $[I ; J] \in \mathbf{N}(k)$ and a singular e(k)-monomial otherwise. For an $\varepsilon(k)$-monomial $e_{[I ; J]}$, the notation $\varepsilon_{[I ; J]}$ is used as well. 
Keeping in mind the correspondence between marked $k$-partitions and $e(k)$-monomials, we shall apply the notions related to such partitions (degree, length, etc.) to $e(k)$-monomials. Note that $e_{[I ; J]}=0$ may be zero for a nonempty $[I ; J]$. For example, $e_{2} \wedge \delta_{1} e_{3}=0$. This motivates the following definition.

Definition 2.2. We write $e_{[I ; J]} \triangleleft e_{\left[I^{\prime} ; J^{\prime}\right]}$ if $e_{\left[I^{\prime} ; J^{\prime}\right]} \neq 0$ and ether $e_{[I ; J]}=0$ or $[I ; J] \triangleleft\left[I^{\prime} ; J^{\prime}\right]$.

Corollary 1.7 implies the following assertion.

Lemma 2.3. If $e_{[I ; J]} \Downarrow e_{\left[I^{\prime} ; J^{\prime}\right]}$ and $e_{\left[I_{1} ; J_{1}\right]} \triangleleft e_{\left[I_{1}^{\prime} ; J_{1}^{\prime}\right]}$, then $e_{[I ; J]} \wedge e_{\left[I_{1} ; J_{1}\right]} \triangleleft e_{\left[I^{\prime} ; J^{\prime}\right]} \wedge e_{\left[I_{1}^{\prime} ; J_{1}^{\prime}\right]}$.

Theorem 2.4. The set of $\varepsilon(k)$-monomials is a basis of the space $C^{*}(k)$ and has the property that

$$
\begin{aligned}
e_{[I ; J]} & =\sum_{\left[I^{\prime} ; J^{\prime}\right] \triangleleft[I ; J]} \alpha_{\left[I^{\prime} ; J^{\prime}\right]} \varepsilon_{\left[I^{\prime} ; J^{\prime}\right]} \quad \text { if }[I ; J] \text { is a singular marked k-partition, } \\
\delta_{k}\left(\varepsilon_{I}\right) & =\alpha_{I} \varepsilon_{\left[I ; I^{-}\right]}+\sum_{\left[I^{\prime} ; J^{\prime}\right], I^{\prime} \triangleleft I} \alpha_{\left[I^{\prime} ; J^{\prime}\right]} \varepsilon_{\left[I^{\prime} ; J^{\prime}\right]} \quad\left(\alpha_{I} \neq 0\right) \quad \text { if } I \text { is a dense k-partition. } .
\end{aligned}
$$

Formula (9) implies that for a dense or main $k$-partition $I$ we have

$$
\delta_{k}\left(\varepsilon_{\left[I ; I^{-}\right]}\right)=\sum_{\left[I^{\prime} ; J^{\prime}\right], I^{\prime} \triangleleft I} \alpha_{\left[I^{\prime} ; J^{\prime}\right]} \varepsilon_{\left[I^{\prime} ; J^{\prime}\right]} \quad \text { or } \quad \delta_{k}\left(\varepsilon_{I}\right)=\sum_{\left[I^{\prime} ; J^{\prime}\right], I^{\prime} \triangleleft I, \operatorname{ind}_{k} I^{\prime}>0} \alpha_{\left[I^{\prime} ; J^{\prime}\right]} \varepsilon_{\left[I^{\prime} ; J^{\prime}\right]},
$$

respectively.

Let us apply Theorem 2.4 to compute $H^{*}(k)=H^{*}(L(k) ; \mathbb{Q})$. Let $\mathrm{N}_{0}(k)=\mathrm{N}(k)$ and

$$
\mathrm{N}_{t+1}(k)=\mathrm{N}_{t}(k) \backslash\left\{\text { the set of maximal elements in } \mathrm{N}_{t}(k) \text { with respect to } ₫\right\} \text {, }
$$

where $t \in \mathbb{Z}_{\geqslant 0}$. Let $\pi: \mathbf{N}(k) \rightarrow \mathrm{N}(k)$ be the projection defined by $\pi[I ; J]=I$, and let $E_{t}(k)$ be the linear span of the set of $\varepsilon(k)$-monomials $\varepsilon_{[I ; J]}$, where $[I ; J] \in \pi^{-1}\left(\mathrm{~N}_{t}(k)\right)$. By Theorem 2.4, $C^{*}(k)=E_{0}(k)$. Hence we obtain a filtration

$$
C^{*}(k)=E_{0}(k) \supset E_{1}(k) \supset E_{2}(k) \supset \cdots
$$

of vector spaces. Let $\varepsilon_{[I ; J]} \in E_{t}(k)$, and let $I_{1} \sqcup \cdots \sqcup I_{m}$ be the standard form of the $k$-partition $I$. By formula (8),

$$
\begin{aligned}
\delta_{k}\left(\varepsilon_{[I ; J]}\right) & =\delta_{k}\left(\varepsilon_{\left[I_{1} ; I_{1} \cap J\right]} \wedge \cdots \wedge \varepsilon_{\left[I_{s} ; I_{s} \cap J\right]}\right) \\
& =\sum_{1 \leqslant a \leqslant s}(-1)^{\eta_{a}(I ; J)} \varepsilon_{\left[I_{1} ; I_{1} \cap J\right]} \wedge \cdots \wedge \delta_{k} \varepsilon_{\left[I_{a} ; I_{a} \cap J\right]} \wedge \cdots \wedge \varepsilon_{\left[I_{s} ; I_{s} \cap J\right]},
\end{aligned}
$$

where $\eta_{1}(I ; J)=1$ and $\eta_{a}(I ; J)=\left|I_{1}\right|+\cdots+\left|I_{a-1}\right|+\left|I_{1} \cap J\right|+\cdots+\left|I_{a-1} \cap J\right|$ for $a>1$. Formulas (10) and (11) imply that $\delta_{k} E_{t}(k) \subset E_{t}(k)$. Therefore, (12) is a filtration of the complex $\left\{C^{*}(k), \delta_{k}\right\}$.

Let $\left\{\mathscr{E}_{r}(k), \partial_{r}\right\}$ be the $r$ th page of the corresponding spectral sequence. The image of an $\varepsilon(k)$ monomial $\varepsilon_{[I ; J]}$ under the natural projection $C^{*}(k) \rightarrow \mathscr{E}_{0}(k)$ will be denoted by the same symbol. The differential $\partial_{0}$ acts by formula (13) with $\delta_{k}$ replaced by $\partial_{0}$.

For $I_{a} \in \mathrm{M}(k)$, it follows from the second formula in (11) and Lemma 2.3 that the corresponding term on the right-hand side in (13) belongs to $E_{t+1}(k)$. The same is true for $I_{a}^{-} \in J$, as follows from the first formula in (11) and Lemma 2.3. In particular, for a main or dense $k$-partition $I$ we have

$$
\partial_{0} \varepsilon_{[I ; J]}= \begin{cases}0 & \text { if }|J|=1 \text { or } \operatorname{ind}_{k} I=0 \\ \alpha_{I} \varepsilon_{\left[I ; I^{-}\right]} \neq 0 & \text { if }|J|=0 \text { and } \operatorname{ind}_{k} I=1 .\end{cases}
$$

Let $C(I) \subset \mathscr{E}_{0}(k)$ be the space spanned by all chains $\varepsilon_{[I ; J]}$ with $I$ fixed. Then formula (14) shows that $\mathscr{E}_{0}(k)=\bigoplus_{I \in \mathrm{N}(k)} C(I)$ (a direct sum of complexes).

It follows from (9), (10), and the definition of spectral sequence that $H^{*}(C(I)) \cong \mathbb{Q} \varepsilon_{I}$ if $I$ is a main $k$-partition and $H^{*}(C(I))=0$ if $I$ is a dense $k$-partition. 
Formula (13) shows that $C(I)=\bigotimes_{1 \leqslant s \leqslant m} C\left(I_{s}\right)$ is a tensor product of complexes. Therefore, by the Künneth formula,

$$
H^{*}(C(I)) \cong \begin{cases}0 & \text { if } \operatorname{ind}_{k} I \neq 0 \\ \mathbb{Q} \varepsilon_{I} & \text { if } \operatorname{ind}_{k} I=0 .\end{cases}
$$

Thus, $\mathscr{E}_{1}(k)=\bigoplus_{I \in \mathrm{M}_{q}(k)} \mathbb{Q} \varepsilon_{I}$. Now it follows from (14) that $\partial_{1}=\partial_{2}=\cdots=0$. Hence $\mathscr{E}_{\infty}(k)=$ $\mathscr{E}_{1}(k)$. The calculation is complete.

Theorem 2.5. For any $I \in \mathrm{M}_{q}(k)$, there exists a cocycle

$$
\mathscr{C}_{I}=\varepsilon_{I}+\sum_{\left[I^{\prime} ; J^{\prime}\right] \triangleleft I, \operatorname{ind}_{k} I^{\prime}>0} \alpha_{\left[I^{\prime} ; J^{\prime}\right]} \varepsilon_{\left[I^{\prime} ; J^{\prime}\right]} \in C^{q}(k)
$$

representing a nonzero class $\mathscr{C}_{I} \in H^{q}(k)$. The set of homology classes of the cocycles $\mathscr{C}_{I}$ is a basis of the space $H^{q}(k)$.

Remark 2.6. Theorem 2.5 shows that formula (3) is equivalent to the formula (see [7])

$$
\left|\mathrm{M}_{q}(k)\right|=\left(\begin{array}{c}
q+k-1 \\
k-1
\end{array}\right)+\left(\begin{array}{c}
q+k-2 \\
k-1
\end{array}\right),
$$

which can readily be verified by induction on $k$.

Remark 2.7. From Theorems 2.4 and 2.5, one can get some information on the multiplication induced in $H^{*}(k)$ by the exterior multiplication of cochains. Note that $\psi(k,|I|) \leqslant\|I\| \leqslant \psi(2 k,|I|)$ for $I \in \mathrm{M}_{q}(k)$ and $\psi(k, q)=k q+3 q(q-1) / 2$.

Assume that $I_{1}, I_{2} \in \mathrm{M}(k)$ and $\mathscr{C}_{I_{1}} \wedge \mathscr{C}_{I_{2}}$ represents a nonzero class $\mathscr{C}_{I_{1}} \cdot \mathscr{C}_{I_{2}} \in H^{*}(k)$. Then, by formulas (15) and (9) and Lemma 2.3, there exists an $I \in \mathrm{M}_{\left|I_{1}\right|+\left|I_{2}\right|}(k)$ with $I \Vdash I_{1} \sqcup I_{2}$. Therefore, $\psi\left(k,\left|I_{1}\right|+\left|I_{2}\right|\right) \leqslant\left\|I_{1}\right\|+\left\|I_{2}\right\| \leqslant \psi\left(2 k,\left|I_{1}\right|\right)+\psi\left(2 k,\left|I_{2}\right|\right)$. This implies the condition $3\left|I_{1}\right|\left|I_{2}\right| \leqslant k\left(\left|I_{1}\right|+\left|I_{2}\right|\right)$ necessary for $\mathscr{C}_{I_{1}} \cdot \mathscr{C}_{I_{2}}$ to be nonzero. It follows from this condition that the multiplication in the cohomology of $L(1)$ and $L(2)$ is trivial.

If $I_{1}, I_{2}, I_{1} \sqcup I_{2} \in \mathrm{M}(k)$, then $\mathscr{C}_{I_{1}} \cdot \mathscr{C}_{I_{2}}=\operatorname{sg}\left(I_{1}, I_{2}\right) \mathscr{C}_{I_{1} \sqcup I_{2}}$, where $\operatorname{sg}\left(I_{1}, I_{2}\right)$ is the sign of the permutation $\left(\begin{array}{c}I_{1} \sqcup I_{2} \\ I_{1}, I_{2}\end{array}\right)$. Hence the multiplication in $H^{*}(k)$ is nontrivial for $k>2$. Theorems 2.4 and 2.5 reduce computing the multiplication in $H^{*}(k)$ to computing the coefficients $\alpha_{I}$ in the expansion

$$
\varepsilon_{I_{1}} \wedge \varepsilon_{I_{2}}=\sum_{I \triangleleft I_{1} \sqcup I_{2}} \alpha_{I} \varepsilon_{I}+\sum_{\left[I^{\prime} ; J^{\prime}\right] \triangleleft I_{1} \sqcup I_{2}, \text { ind }_{k} I^{\prime}>0} \beta_{\left[I^{\prime} ; J^{\prime}\right]} \varepsilon_{\left[I^{\prime} ; J^{\prime}\right]}
$$

where $I_{1}, I_{2}, I \in \mathrm{M}(k)$. To derive a general formula for these coefficients is apparently a difficult combinatorial problem.

\section{Proof of (Main) Theorem 2.4}

Lemma 3.1. In the complex $C^{*}(k)$, we have

$$
\begin{gathered}
\sum_{a+b=n}(a)_{h} e_{a} \wedge \delta_{k}\left(e_{b}\right)=0, \\
\sum_{a+b=n} \delta_{k}\left(e_{a}\right) \wedge \delta_{k}\left(e_{b}\right)=0, \quad \sum_{a+b=n}(b-a)_{h}^{2} \delta_{k}\left(e_{a}\right) \wedge \delta_{k}\left(e_{b}\right)=0 .
\end{gathered}
$$

If either $h=1$ or $h \neq 1$ and $n \not \equiv 0 \bmod 3$, then

$$
\sum_{a+b=n} e_{a} \wedge \delta_{k}\left(e_{b}\right)=0, \quad \sum_{a+b=n}(a)_{h}(b-a)_{h}^{2} e_{a} \wedge \delta_{k}\left(e_{b}\right)=0 .
$$

If $h \neq 1$ and $n \equiv 0 \bmod 3$, then

$$
\sum_{a+b=n}(n-3 a) e_{a} \wedge \delta_{k}\left(e_{b}\right)=0, \quad \sum_{a+b=n}\left(2-3(a)_{h}^{2}\right) e_{a} \wedge \delta_{k}\left(e_{b}\right)=0
$$


Proof. The relation $\sum_{a+b=n} f(a, b) e_{a} \wedge \delta_{k}\left(e_{b}\right)=0$ is equivalent to the identity

$$
(y-z)_{h} f(x, y+z)-(x-z)_{h} f(y, x+z)+(x-y)_{h} f(z, x+y)=0 .
$$

A straightforward verification shows that this identity holds for (16), (18), and (19). By applying $\delta_{k}$ to $(18)$ or (19), we obtain (17).

Definition 3.2. An $e(k)$-monomial $e_{[I ; J]}$ is said to be $\operatorname{good}$ if the $k$-partition $[I ; J]$ is nonsingular as a marked 1-partition and bad otherwise.

Lemma 3.3. For any bad e(k)-monomial $e_{[I ; J]} \in C^{*}(k)$, there exists a decomposition

$$
e_{[I ; J]}=\sum_{\left[I^{\prime} ; J^{\prime}\right] \triangleleft[I ; J]} \alpha_{\left[I^{\prime} ; J^{\prime}\right]} e_{\left[I^{\prime} ; J^{\prime}\right]}
$$

where all $e(k)$-monomials $e_{\left[I^{\prime} ; J^{\prime}\right]}$ are good.

Proof. Obviously, an $e(k)$-monomial is bad if and only if it contains length 2 submonomials from the following list, where $\alpha=0,1$ :

$$
\begin{gathered}
x(i, \alpha)=e_{i-1+\alpha} \wedge e_{i+1}, \\
y_{1}(i, \alpha)=e_{i} \wedge \delta_{k} e_{i+\alpha}, \quad y_{2}(i, \alpha)=\delta_{k} e_{i-1+\alpha} \wedge e_{i+1}, \quad y_{3}(i, \alpha)=e_{i-1} \wedge \delta_{k} e_{i+1+\alpha}, \\
z_{1}(i, \alpha)=\delta_{k} e_{i} \wedge \delta_{k} e_{i+\alpha}, \quad z_{2}(i, \alpha)=\delta_{k} e_{i-1} \wedge \delta_{k} e_{i+1+\alpha} .
\end{gathered}
$$

Let $|I|=2$. Consider (7) as an equation for the unknown $x(i, \alpha)$; identities (16) and (18) for $h=1$ or $h \neq 1$ and $n \neq \equiv 0 \bmod 3$, or identities (16) and (19) for $h \neq 1$ and $n \equiv 0 \bmod 3$ as a system of equations for the unknowns $y_{1}(i, \alpha), y_{2}(i, \alpha)$, and $y_{3}(i, \alpha)$; and finally (17) as a system of equations for the unknowns $z_{1}(i, \alpha)$ and $z_{2}(i, \alpha)$. A straightforward verification shows that these systems of linear equations are nonsingular. Their solutions give the decomposition (20) for bad $e(k)$-monomials of length 2 .

For $|I|>2$, let us replace an arbitrary (e.g., the leftmost) bad $e(k)$-submonomial of length 2 in $e_{[I ; J]}$ by a linear combination of good ones. Then $e_{[I ; J]}$ will be expressed as a linear combination of $e(k)$-monomials each of which is less than $e_{[I ; J]}$ by Lemma 2.3. Let us apply the same procedure to each bad term in the resulting sum, etc. Since the number of marked partitions $\left[I^{\prime} ; J^{\prime}\right] \triangleleft[I ; J]$ is finite, we arrive at the decomposition (20) in finitely many steps.

Definition 3.4. For $c=\sum \alpha_{[I ; J]} e_{[I ; J]} \in C^{*}(k)$, we write $c \approx 0$ if $c=\sum \alpha_{\left[I^{\prime} ; J^{\prime}\right]} e_{\left[I^{\prime} ; J^{\prime}\right]}$ and $\left[I^{\prime} ; J^{\prime}\right] \triangleleft[I ; J]$ for all pairs $[I ; J]$ and $\left[I^{\prime} ; J^{\prime}\right]$ with $\alpha_{[I ; J]} \alpha_{\left[I^{\prime} ; J^{\prime}\right]} \neq 0$. For $c_{1}, c_{2} \in C^{*}(k)$, we write $c_{1} \approx c_{2}$ if $c_{1}-c_{2} \approx 0$.

Lemma 3.5. Let $I$ be a dense $k$-partition. Then $\delta_{k}\left(\varepsilon_{I}\right) \approx \alpha_{I} \varepsilon_{\left[I ; I^{-}\right]}$, where $\alpha_{I} \neq 0$.

Proof. By applying the algorithm of Lemma 3.3 to $I$, we obtain

$$
e_{\left[I ; I^{+}\right]} \approx \begin{cases}(-1)^{|I|-1} I^{+} / I^{-} \varepsilon_{\left[I ; I^{-}\right]} & \text {if } h=1 \text { or } h \neq 1 \text { and } n \neq 0 \bmod 3, \\ (-1)^{|I|-1} \varepsilon_{\left[I ; I^{-}\right]} & \text {if } h \neq 1 \text { and } n \equiv 0 \bmod 3 .\end{cases}
$$

If $I=\left[i_{1}, \ldots, i_{q}\right]$ and $I_{r}=\left[i_{1}, \ldots, i_{r}\right]$, then

$$
\delta_{k}\left(\varepsilon_{I}\right)=\sum_{1 \leqslant r \leqslant q}(-1)^{r-1} e_{\left[I_{r} ; I_{r}^{+}\right]} \wedge \varepsilon_{I \backslash I_{r}} .
$$

By substituting the expressions (21) for $e_{\left[I_{r} ; I_{r}^{+}\right]}$into this formula, we obtain

$$
\delta_{k}\left(\varepsilon_{I}\right) \approx \begin{cases}\|I\| / I^{-} \varepsilon_{\left[I ; I^{-}\right]}, & \text {if } h=1 \text { or } h \neq 1 \text { and } n \neq \equiv 0 \bmod 3, \\ |I| \varepsilon_{\left[I ; I^{-}\right]}, & \text {if } h \neq 1 \text { and } n \equiv 0 \bmod 3 .\end{cases}
$$

This completes the proof.

The sets of good $e(1)$-monomials and $\varepsilon(1)$-monomials coincide. Hence Lemmas 3.3 and 3.5 imply that the set of $\varepsilon(1)$-monomials of degree $n$ is a linear system of generators of the vector 
space $C_{*}^{(n)}(1)$, for which formulas (9) and (10) hold. To finish the proof of Theorem 2.4 for $k=1$, it suffices to show that the $\varepsilon(1)$-monomials are linearly independent.

Let $\mathbf{N}_{l, m}^{(n)}=\{[I ; J] \in \mathbf{N}(1):\|I\|=n,|I|=l,|J|=m\}$. The number of $\varepsilon(1)$-monomials of dimension $q$ and degree $n$ is equal to the cardinality of the set $\mathbf{N}_{q}^{(n)}=\bigsqcup_{l+m=q} \mathbf{N}_{l, m}^{(n)}$. On the other hand, $\operatorname{dim} C_{(n)}^{q}(1)=\left|\mathrm{D}_{q}^{(n)}\right|$, where $\mathrm{D}_{q}^{(n)}$ is the set of strict 1-partitions of length $q$ and degree $n$. Therefore, the linear independence of $\varepsilon(1)$-monomials is a consequence of the following claim:

Theorem 3.6. $\left|\mathrm{D}_{q}^{(n)}\right|=\left|\mathbf{N}_{q}^{(n)}\right|$.

It suffices to show that

Lemma 3.7. The claim of Theorem 3.6 and identity (4) are equivalent.

Proof. Let $A_{l}(x, t)=\sum_{n=1}^{\infty} \sum_{m=0}^{l}\left|\mathbf{N}_{l, m}^{(n)}\right| t^{m} x^{n}$. One can readily see that Theorem 3.6 is equivalent to the identity

$$
\prod_{i=1}^{\infty}\left(1+t x^{i}\right)=1+\sum_{n=1}^{\infty} \sum_{q=1}^{\infty}\left|\mathbf{N}_{q}^{(n)}(1)\right| t^{q} x^{n}=1+\sum_{l=1}^{\infty} A_{l}(x, t) t^{l}
$$

Now it suffices to verify that

$$
A_{l}(x, t)=x^{\left(3 l^{2}-l\right) / 2} \frac{(1+t x) \cdots\left(1+t x^{l-1}\right)\left(1+t x^{2 l}\right)}{(1-x) \cdots\left(1-x^{l-1}\right)\left(1-x^{l}\right)} .
$$

The property of a 1 -partition $\left[i_{1}, \ldots, i_{l}\right]$ to be nonsingular is equivalent to the following property of the dual partition (see [10]): it is a partition in which $i_{1} \geqslant 1$ parts are equal to $l, i_{2}-i_{1} \geqslant 3$ parts are equal to $l-1, \ldots, i_{l}-i_{l-1} \geqslant 3$ parts are equal to 1 . If either $i_{1} \geqslant 3$ or $i_{a}-i_{a-1}>3$ for some $a$, where $2 \leqslant a \leqslant q$, then the corresponding part $\left(i_{1}\right.$ or $\left.i_{a}\right)$ of the partition can be either marked (the coefficient $t$ ) or not (the coefficient 1 ). Thus,

$$
A_{1, l}(x, t)=\left(x^{l}+x^{2 l}+(1+t) \sum_{r=3}^{\infty} x^{r l}\right)\left(x^{3(l-1)}+(1+t) \sum_{r=4}^{\infty} x^{r(l-1)}\right) \cdots\left(x^{3}+(1+t) \sum_{r=4}^{\infty} x^{r}\right) .
$$

By summing the geometric progressions in the parentheses, we arrive at the desired formula.

The proof of Theorem 2.4 for $k=1$ is complete. For $k>1$, we shall prove it by induction on $k$. First, we introduce two useful operations on marked partitions.

Definition 3.8. Let $\sigma\left[i_{1}, \ldots, i_{q}\right]=\left[i_{1}+1, \ldots, i_{q}+1\right]$. Define a mapping $\sigma: \mathbf{D}(k) \rightarrow \mathbf{D}(k+1)$ by the formula $\sigma[I ; J]=\left[\sigma(I \backslash J) \sqcup \sigma^{2}(J) ; \sigma^{2}(J)\right]$.

Definition 3.9. Let $[I ; J] \in \mathbf{N}(k)$, let $I_{1} \sqcup \cdots \sqcup I_{m}$ be the standard form of the $k$-partition $I$, and let $J=\left[I_{a_{1}}^{-}, \ldots, I_{a_{r}}^{-}\right]$. Define $\tau[I ; J]=\left[I ; J^{+}\right] \in \mathbf{D}(k)$, where $J^{+}=\left[I_{a_{1}}^{+}, \ldots, I_{a_{r}}^{+}\right]$.

The definitions readily imply that

Lemma 3.10. The composition $\sigma \tau$ induces a bijective mapping $\sigma \tau: \mathbf{N}(k) \rightarrow \mathbf{N}(k+1)$.

Assume that Theorem 2.4 is true for $\varepsilon(k-1)$-monomials. Then the set of monomials $e_{\tau[I ; J]}$, where $[I ; J] \in \mathbf{N}(k-1)$, is a basis of $C^{*}(k-1)$, and

$$
\varepsilon_{[I ; J]}=\beta_{[I ; J]} e_{\tau[I ; J]}+\sum_{\left[I^{\prime} ; J^{\prime}\right] \triangleleft[I ; J]} \beta_{\left[I^{\prime} ; J^{\prime}\right]} e_{\tau\left[I^{\prime} ; J^{\prime}\right]}, \quad \text { where } \beta_{[I ; J]} \neq 0 .
$$

Indeed, formula (21) and Lemma 2.3 show that

$$
e_{\tau[I ; J]} \approx \gamma\left(I_{a_{1}}\right) \cdots \gamma\left(I_{a_{1}}\right) \varepsilon_{[I ; J]} \neq 0,
$$

where $I_{1} \sqcup \cdots \sqcup I_{m}$ is the standard form of the $(k-1)$-partition $I, J=\left[I_{a_{1}}^{-}, \ldots, I_{a_{r}}^{-}\right]$, and $\gamma\left(I_{a}\right)$ is the coefficient of $\varepsilon_{\left[I_{a} ; I_{a}^{-}\right]}$in formula (21). Hence, under the linear ordering of $\mathbf{N}(k-1)$ compatible with $\sharp$, the subset of $e(k-1)$-monomials $e_{\tau[I ; J]}$ of fixed degree can be expressed via the basis of $\varepsilon(k-1)$-monomials of the same degree by an invertible upper triangular matrix. By inverting 
this matrix, we see that the $e(k-1)$-monomials $e_{\tau[I ; J]}$ form a basis of the space $C^{*}(k-1)$ as well and that formula (22) holds for this basis, since the inequality $\tau\left[I^{\prime} ; J^{\prime}\right] \triangleleft[I ; J]$ implies that $\left[I^{\prime} ; J^{\prime}\right] \triangleleft[I ; J]$.

Let $\sigma: C^{*}(k-1) \rightarrow C^{*}(k)$ be the linear isomorphism defined by the formula $\sigma\left(e_{I}\right)=e_{\sigma(I)}$. Since $\sigma \delta_{k}\left(e_{i}\right)=\delta_{k+1}\left(e_{i+2}\right)$, we have $\sigma\left(e_{[I ; J]}\right)=e_{\sigma[I ; J]}$. By applying $\sigma$ to both sides of (22), we obtain

$$
\sigma\left(\varepsilon_{[I ; J]}\right)=\beta_{[I ; J]} \varepsilon_{\sigma \tau[I ; J]}+\sum_{\left[I^{\prime} ; J^{\prime}\right] \triangleleft[I ; J]} \beta_{\left[I^{\prime} ; J^{\prime}\right]} \varepsilon_{\sigma \tau\left[I^{\prime} ; J^{\prime}\right]}, \quad \text { where } \beta_{[I ; J]} \neq 0,
$$

since $e_{\sigma \tau\left[I^{\prime} ; J^{\prime}\right]}=\varepsilon_{\sigma \tau\left[I^{\prime} ; J^{\prime}\right]}$ by Lemma 3.10 .

By the induction assumption, the set of chains $\sigma\left(\varepsilon_{[I ; J]}\right)$ is a basis of $C^{*}(k)$, since $\sigma$ is an isomorphism. On the other hand, the set of chains $\varepsilon_{\sigma \tau[I ; J]}$ coincides with the set of $\varepsilon(k)$-monomials by Lemma 3.10. As earlier, Eq. (23) implies that these sets are related by an invertible triangular transformation. Thus, the $\varepsilon(k)$-monomials form a basis of $C^{*}(k)$ as well. Now formula (10) follows from Lemma 3.5. It remains to prove formula (9).

Note that a good $e(k)$-monomial $e_{[I ; J]}$ is not an $\varepsilon(k)$-monomial if and only if the main component of the nonsingular $k$-partition $I$ is not empty and contains marked parts. If such $e(k)$ monomials are absent in the decomposition (20), then the decomposition coincides with (9).

An algorithm similar to that used in the proof of Lemma 3.3 shows that it suffices to consider the case in which $I=\left[i_{1}, \ldots, i_{q}\right] \in \mathrm{M}(k), J=\left[i_{q}\right]$, and $i_{q}-i_{q-1}>3$. Then $\sigma^{-1}\left[I ; I^{+}\right]=\left[I_{1} ; I_{1}^{+}\right]$, where $I_{1} \in \mathrm{M}(k-1)$. By the induction assumption,

$$
\sigma^{-1}\left(e_{\left[I ; I^{+}\right]}\right)=\sum_{\left[I^{\prime} ; J^{\prime}\right] \triangleleft \sigma^{-1}\left[I ; I^{+}\right],\left[I^{\prime} ; J^{\prime}\right] \in \mathbf{N}(k-1)} \alpha_{\left[I^{\prime} ; J^{\prime}\right]} e_{\tau\left[I^{\prime} ; J^{\prime}\right]} .
$$

An application of the operator $\sigma$ to this formula gives the decomposition (9) for $e_{\left[I ; I^{+}\right]}$, since the following claim is true:

Lemma 3.11. Let $I \in \mathrm{M}(k),\left[I^{\prime} ; J^{\prime}\right] \in \mathbf{N}(k)$, and $\left[I^{\prime} ; J^{\prime}\right] \triangleleft\left[I ; I^{+}\right]$. Then $\sigma \tau\left[I^{\prime} ; J^{\prime}\right] \triangleleft \sigma\left[I ; I^{+}\right]$.

Proof. The claim is nontrivial only for $\left|I^{\prime}\right|=|I|$. Let $I^{\prime}=I_{1}^{\prime} \sqcup \cdots \sqcup I_{s}^{\prime}$ be the standard form, and let $J^{\prime}=\left[I_{l}^{\prime-}\right]$. Since $I^{\prime} \triangleleft I$ and $I^{\prime}$ is nonsingular, it follows that $I_{1}^{\prime} \neq \varnothing$ is a main $k$-partition and $I_{2}^{\prime} \neq \varnothing$. Let $|I|=q$ and $I_{l}^{\prime+}=i_{p}^{\prime}$. For $p=q$, the claim is obvious. Assume that $p<q$.

It suffices to show that inequalities (5) are strict for all $r \geqslant p$. Indeed, if $r \geqslant p$, then $\sigma \tau\left[I^{\prime} ; J^{\prime}\right] \triangleleft$ $\sigma\left[I ; I^{+}\right]$. But $\sigma\left[I ; I^{+}\right] \notin \mathbf{N}(k+1)$, while $\sigma \tau\left[I^{\prime} ; J^{\prime}\right] \in \mathbf{N}(k+1)$ by Lemma 3.10. Hence $\sigma \tau\left[I^{\prime} ; J^{\prime}\right] \triangleleft$ $\sigma\left[I ; I^{+}\right]$.

For $p \leqslant r \leqslant q$, the definitions imply that

$$
\begin{array}{ll}
i_{r}<2 k+3(r-1) \leqslant i_{r}^{\prime} & \text { if } i_{1}=k, \\
i_{r} \leqslant 2 k+3(r-1)<i_{r}^{\prime} & \text { if } i_{1}>k \text { and } i_{1}^{\prime}>k, \\
i_{r} \leqslant 2 k+3(r-1) \leqslant i_{r}^{\prime} & \text { if } i_{1}>k \text { and } i_{1}^{\prime}=k .
\end{array}
$$

Assume first that $i_{r}^{\prime}>i_{r}$ for all $r \geqslant p$. Then the minimum $a$ such that $p \leqslant a \leqslant q$ and $i_{1}^{\prime}+\cdots+i_{a}^{\prime}=$ $i_{1}+\cdots+i_{a}$ is equal to $q$. Hence inequalities (5) are strict for $r \geqslant p$.

Suppose that there exists an $r \geqslant p$ with $i_{r}^{\prime}=i_{r}$, and let $r_{0}$ be the maximum $r$ with this property. It follows from (24) that $i_{r_{0}}^{\prime}=i_{r_{0}}=2 k+3\left(r_{0}-1\right)$. Hence $l=2$ and $p=r_{0}$. Since $i_{p}^{\prime}=i_{p}$ and $i_{r}^{\prime}>i_{r}$ for $r>p$, we can argue as before to see that inequalities (5) are strict for $r \geqslant p$.

\section{Stable Filtration of the Complex $C_{*}(k)$}

In this section, we treat $e(k)$-monomials as vectors in the space $C_{*}(k)$.

Definition 4.1. Let $\mathrm{St}_{0}(k)=0$ and

$$
\mathrm{St}_{m}(k)=\left\{c \in C_{*}(k): d_{k+r} \sigma^{r}(c) \in \mathrm{St}_{m-1}(k+r) \text { for all } r \geqslant 0\right\}
$$


for any integer $m>0$. The vectors in the space $\operatorname{St}_{m}(k)$ are called $m$-stable chains, and the filtration

$$
0=\operatorname{St}_{0}(k) \subset \operatorname{St}_{1}(k) \subset \operatorname{St}_{2}(k) \subset \cdots \subset \operatorname{St}_{\infty}(k)=C_{*}(k)
$$

is called the stable filtration of $C_{*}(k)$. A stable cycle is a 1-stable chain (see [7]).

Definition 4.2. Let $c=\sum \alpha_{[I ; J]} \varepsilon_{[I ; J]}$ be the expansion of $c \in C_{*}(k)$ in the basis of $\varepsilon(k)$ monomials. Let $\operatorname{ht}_{k}(c)=\min \left\{|J|: \alpha_{[I ; J]} \neq 0\right\}$ and $h_{k}(0)=\infty$. Denote by $\mathrm{E}_{m}(k)=\mathrm{E}_{m}(L(k))$ the space of chains $c \in C_{*}(k)$ with $\mathrm{ht}_{k}(c) \geqslant m$. The filtration

$$
C_{*}(k)=\mathrm{E}_{0}(k) \supset \mathrm{E}_{1}(k) \supset \mathrm{E}_{2}(k) \supset \cdots \supset \mathrm{E}_{\infty}(k)=0
$$

is called the $\varepsilon$-filtration of $C_{*}(k)$.

Theorem 4.3. $\left.\operatorname{St}_{m}(k)=\widehat{\mathrm{E}}_{m}(k):=\left\{c \in C^{(} k\right):\left\langle c, \mathrm{E}_{m}(k)\right\rangle=0\right\}$.

Lemma 4.4. If $m \geqslant 1$, then for any $c \in \mathrm{E}_{m}(k)$ and $r \gg 0$ there exists a chain $c_{r} \in \mathrm{E}_{m-1}(k+r)$ such that $\sigma^{r}(c)=\delta_{k+r}\left(c_{r}\right)$.

Proof. We say that a partition of the form $[I ; J]=\left[i_{1}, \ldots, i_{p}, i_{p+1}, \ldots, i_{q}\right] \in \mathbf{N}(k)$, where $i_{p} \leqslant 2 k$, is stable. For stable $[I ; J]$, the following formula proves the lemma for $\bar{c}=\varepsilon_{[I ; J]}$ :

$$
\varepsilon_{[I ; J]}=\varepsilon_{\left[i_{1}, \ldots, i_{p}\right]} \wedge \varepsilon_{\left[\underline{i_{p+1}}, \ldots, i_{q}\right]}=\delta_{k}\left(\varepsilon_{\left[i_{1}, \ldots, i_{p}\right]} \wedge \varepsilon_{\left[i_{p+1}, \underline{i_{p+2}}, \ldots, i_{q}\right]}\right) .
$$

For arbitrary $\left[I^{\prime} ; J^{\prime}\right] \in \mathbf{N}(k)$, there exists an $r\left(I^{\prime} ; J^{\prime}\right) \gg 0$ such that $(\sigma \tau)^{r\left(I^{\prime} ; J^{\prime}\right)}\left[I^{\prime} ; J^{\prime}\right]$ is stable. But then there exists an $r(I ; J)$ such that $(\sigma \tau)^{r(I ; J)}\left[I^{\prime} ; J^{\prime}\right]$ is stable for all $\left[I^{\prime} ; J^{\prime}\right] \in \mathbf{N}(k)$ with $\left[I^{\prime} ; J^{\prime}\right] \preccurlyeq[I ; J]$, because the number of such $\left[I^{\prime} ; J^{\prime}\right]$ is finite.

It follows from $(23)$ by induction on $r$ that

$$
\sigma^{r}\left(\varepsilon_{[I ; J]}\right)=\beta_{r,[I ; J]} \varepsilon_{(\sigma \tau)^{r}[I ; J]}+\sum_{\left[I^{\prime} ; J^{\prime}\right] \triangleleft[I ; J]} \beta_{r,\left[I^{\prime} ; J^{\prime}\right]} \varepsilon_{(\sigma \tau)^{r}\left[I^{\prime} ; J^{\prime}\right]}, \quad \text { where } \beta_{r,[I ; J]} \neq 0,
$$

for $r>0$. Let $c=\sum_{[I ; J]} \alpha_{[I ; J]} \varepsilon_{[I ; J]}$ and $r(c)=\max \left\{r(I ; J): \alpha_{[I ; J]} \neq 0\right\}$. By applying $\sigma^{r(c)}$ to $c$, we obtain Lemma 4.4 from formula $(25)$ and the already considered case of stable $[I ; J]$.

Proof of Theorem 4.3. We argue by induction on $m$. For $m=0$, the claim is obvious. Assume that $\operatorname{St}_{p}(k)=\widehat{\mathrm{E}}_{p}(k)$ for $p<m$.

First, let us show that the chain $c \in \widehat{\mathrm{E}}_{m}(k)$ is $m$-stable. It follows from formula (25) that $\sigma^{r}\left(\mathrm{E}_{m}(k)\right)=\mathrm{E}_{m}(k+r)$. Since $\left\langle\sigma^{r}\left(c_{1}\right), \sigma^{r}\left(c_{2}\right)\right\rangle=\left\langle c_{1}, c_{2}\right\rangle$ for arbitrary $c_{1}, c_{2} \in C_{*}(k)$, we have

$$
\left\langle\sigma^{r}(c), \mathrm{E}_{m}(k+r)\right\rangle=\left\langle\sigma^{r}(c), \sigma^{r}\left(\mathrm{E}_{m}(k)\right)\right\rangle=\left\langle c, \mathrm{E}_{m}(k)\right\rangle=0 .
$$

Hence $\sigma^{r}(c) \in \widehat{\mathrm{E}}_{m}(k+r)$. On the other hand, $\delta_{k+r}\left(\mathrm{E}_{m-1}(k+r)\right) \subset \mathrm{E}_{m}(k+r)$ by Theorem 2.4. Now the desired inclusion $d_{k+r} \sigma^{r}(c) \in \mathrm{St}_{m-1}(k)=\widehat{\mathrm{E}}_{m-1}(k+r)$ follows from the identity

$$
\left\langle d_{k+r} \sigma^{r}(c), \mathrm{E}_{m-1}(k+r)\right\rangle=\left\langle\sigma^{r}(c), \delta_{k+r}\left(\mathrm{E}_{m-1}(k+r)\right)\right\rangle=0 .
$$

It remains to show that each $m$-stable chain belongs to $\widehat{\mathrm{E}}_{m}(k)$. Since $C_{*}(k)=\widehat{\mathrm{E}}_{m}(k) \oplus \mathrm{E}_{m+1}(k)$, it suffices to show that for each $c \in \mathrm{E}_{m+1}(k)$ there exists an $r \geqslant 0$ such that $d_{k+r} \sigma^{r}(c) \notin$ $\widehat{\mathrm{E}}_{m-1}(k+r)$.

By Lemma 4.4, there exist $r$ and $c_{r} \in \mathrm{E}_{m}(k+r)$ such that $\sigma^{r}(c)=\delta_{k+r}\left(c_{r}\right)$. Therefore,

$$
\left\langle d_{k+r} \sigma^{r}(c), c_{r}\right\rangle=\left\langle\sigma^{r}(c), \delta_{k+r}\left(c_{r}\right)\right\rangle=\left\langle\sigma^{r}(c), \sigma^{r}(c)\right\rangle \neq 0 .
$$

Thus, $d_{k+r} \sigma^{r}(c) \notin \widehat{\mathrm{E}}_{m}(k+r)$. But then $d_{k+r} \sigma^{r}(c) \notin \widehat{\mathrm{E}}_{m-1}(k+r) \subset \widehat{\mathrm{E}}_{m}(k+r)$.

Corollary 4.5. For any $[I ; J] \in \mathbf{N}(k)$, denote by $\widehat{\varepsilon}_{[I ; J]} \in C_{*}(k)$ the chain such that

$$
\left\langle\widehat{\varepsilon}_{[I ; J]}, \varepsilon_{\left[I^{\prime} ; J^{\prime}\right]}\right\rangle=\delta_{[I ; J],\left[I^{\prime} ; J^{\prime}\right]}
$$

for all $\left[I^{\prime} ; J^{\prime}\right] \in \mathbf{N}(k)$. The set of chains $\widehat{\varepsilon}_{[I ; J]}$ is a basis of $C_{*}(k)$. The chains $\widehat{\varepsilon}_{[I ; J]}$ with $|J| \leqslant m-1$ form a basis of $\mathrm{St}_{m}(k)$. In particular, the chains $\widehat{\varepsilon}_{I}$ with $I \in \mathbf{N}(k)$ form a basis of the subspace of stable cycles in $C_{*}(k)$. 
Proof. The claim on the basis follows from Theorem 2.4 and the nondegeneracy of the inner product. The second claim follows from Theorem 4.3.

\section{Stable Filtration and Skew-Symmetric Polynomials}

Recall several facts on (skew)symmetric polynomials (see [10, Chap. I, Secs. 3, 5]). Let $\operatorname{Sym}_{q}[t]$ be the $\mathbb{Q}$-algebra of symmetric polynomials, and let $\mathrm{Alt}_{q}[t]$ be the vector space of skew-symmetric polynomials with rational coefficients in the variables $t_{1}, \ldots, t_{q}$. Set $\operatorname{Sym}_{0}[t]=\operatorname{Alt}_{0}[t]=\mathbb{Q}$. If there is no room for confusion, we write $f(t)$ instead of $f\left(t_{1}, \ldots, t_{q}\right)$. The polynomials

$$
\Delta_{I}\left(t_{1}, \ldots, t_{q}\right)=\operatorname{det}\left(t_{r}^{i_{m}}\right)_{(r, m=1, \ldots, q)}
$$

corresponding to strict partitions $I=\left[i_{1}, \ldots, i_{q}\right]$ form a basis of $\operatorname{Alt}_{q}[t]$. The multiplication

$$
\Delta_{I}\left(t_{1}, \ldots, t_{q}\right) \wedge \Delta_{I^{\prime}}\left(t_{1}, \ldots, t_{q^{\prime}}\right)=\operatorname{sg}\left(I, I^{\prime}\right) \Delta_{I \sqcup I^{\prime}}\left(t_{1}, \ldots, t_{q+q^{\prime}}\right)
$$

where $\operatorname{sg}\left(I_{1}, I_{2}\right)$ is the sign of the permutation $\left(\begin{array}{c}I_{1} \sqcup I_{2} \\ I_{1}, I_{2}\end{array}\right)$, equips $\operatorname{Alt}[t]=\bigoplus_{q=0}^{\infty} \operatorname{Alt}_{q}[t]$ with the structure of an anticommutative graded $\mathbb{Q}$-algebra.

The Vandermonde determinant is the polynomial $V_{q}(t)=\Delta_{\rho(q)}(t)$, where $\rho(q)=[0,1, \ldots, q-1]$. The symmetric polynomial $S_{I}(t)=\Delta_{I+\rho(q)}(t) / V_{q}(t)$, where $I$ is a partition with $|I|=q$, is called a Schur polynomial. The Schur polynomials form a basis in $\operatorname{Sym}_{q}[t]$. It is known that

$$
S_{I_{1}}(t) S_{I_{2}}(t)=S_{I_{1}+I_{2}}(t)+\sum_{I \triangleright I_{1}+I_{2}} \alpha_{I} S_{I}(t)
$$

where $I_{1}+I_{2}$ is the componentwise sum of partitions and $\alpha_{I} \in \mathbb{Z}$. One can readily show that

$$
V_{q}^{2}(t)=S_{2 \rho(q)}(t)+\sum_{I \triangleright 2 \rho(q)} \beta_{I} S_{I}(t) .
$$

We identify every chain $c=\sum_{I} r_{I} e_{I} \in C_{q}(k)$ with the polynomial $c(t)=\sum_{I} r_{I} \Delta_{I}(t)$.

Lemma 5.1. Let $c \in C_{q}\left(L^{(h)}(k)\right)$ and $q \geqslant 2$. Then

$$
d_{k}(c(t))=\sum_{1 \leqslant a \leqslant q-1}(-1)^{a-1} c_{a}\left(t_{1}, \ldots, t_{q-1} ; h\right),
$$

where $c_{a}\left(t_{1}, \ldots, t_{q-1} ; h\right)=\left(h^{2}-h\right)^{-1} c\left(t_{1}, \ldots, t_{a-1}, h t_{a}, h^{2} t_{a}, t_{a+1}, \ldots, t_{q-1}\right)$.

Proof. It suffices to consider the case in which $c(t)=\Delta_{I}(t)$. For $q=2$, formula (28) directly follows from (1). For $q>2$, it follows by induction on $q$ from the formula of the $d_{k}$-action on $k$-monomials. In the proof, it is convenient to use the formula

$$
t_{1}^{m} \wedge c\left(t_{1}, \ldots, t_{q-1}\right)=\sum_{1 \leqslant r \leqslant q}(-1)^{p-1} t_{r}^{m} c\left(t_{1}, \ldots, \widehat{t_{r}}, \ldots, t_{q}\right),
$$

where $c\left(t_{1}, \ldots, t_{q-1}\right) \in \operatorname{Alt}_{q-1}[t]$. We omit the corresponding routine computation.

Let $\operatorname{Sym}_{m, q}^{(h)}[t] \subset \operatorname{Sym}_{q}[t]$ be the space of polynomials $p(t)$ such that

$$
\mathrm{T}_{s, q}^{(h)}(p(t))=p\left(h t_{1}, \bar{h} t_{1}, \ldots, h t_{s}, \bar{h} t_{s}, t_{s+1}, \ldots, t_{q-s}\right)=0
$$

for all $s, 0 \leqslant s \leqslant m \leqslant\lfloor q / 2\rfloor$. For $c \in C_{q}(k)$, let $\widetilde{c}(t) \in \operatorname{Sym}_{q}[t]$ be the unique polynomial such that $c(t)=\left(t_{1} \cdots t_{q}\right)^{k} V_{q}(t) \widetilde{c}(t)$.

Theorem 5.2. $c \in \operatorname{St}_{m, q}(k)=\operatorname{St}_{m}(k) \cap C_{q}(k)$ if and only if $\widetilde{c}(t) \in \operatorname{Sym}_{m, q}^{(h)}[t]$.

Proof. The operator $\sigma$ acts on $c(t)$ as the multiplication by $t_{1} \cdots t_{q}$. Therefore, it suffices to consider the case of $k=1$. We argue by induction on $m$. For $m=0$, the claim is obvious. Let $m \geqslant 1$. Since $V_{q}(t)=\prod_{q \geqslant i>j \geqslant 1}\left(t_{i}-t_{j}\right)$, we have

$$
c_{a}\left(t_{1}, \ldots, t_{q-1} ; h\right)=\left(t_{1} \cdots t_{q-1}\right)^{k} V_{q-1}(t) R_{a}(t ; h) \varphi_{a}(t ; h),
$$


where $\varphi_{a}(t ; h)=\varphi_{a}\left(t_{1}, \ldots, t_{q-1} ; h\right)=\widetilde{c}\left(t_{1}, \ldots, t_{a-1}, h t_{a}, \bar{h} t_{a}, t_{a+1}, \ldots, t_{q-1}\right)$ and

$$
R_{a}(t ; h)=R_{a}\left(t_{1}, \ldots, t_{q-1} ; h\right)=t_{a} \prod_{1 \leqslant i \leqslant q-1, i \neq a} \frac{\left(h t_{a}-t_{i}\right)\left(\bar{h} t_{a}-t_{i}\right)}{t_{a}-t_{i}} .
$$

For $c \in \mathrm{St}_{m, q}(1)$, let us apply formula $(28)$ to $\sigma^{r}(c) \in C_{q}(r+1)$. After cancelling the common factor $\left(t_{1} \cdots t_{q-1}\right)^{r+1} V_{q-1}(t)$ we see by the induction assumption that $c \in \operatorname{St}_{m, q}(1)$ if and only if

$$
t_{1}^{r-1} R_{1}(t ; h) \varphi_{1}(t ; h)+\cdots+t_{q-1}^{r-1} R_{q-1}(t ; h) \varphi_{q-1}(t ; h)=p_{r}(t ; h) \in \operatorname{Sym}_{m-1, q-1}^{(h)}[t]
$$

for every integer $r \geqslant 1$. For $r=1, \ldots, q-1$, we obtain a system of linear equations for the unknowns $X_{a}(t ; h)=R_{a}(t ; h) \varphi_{a}(t ; h)$.

Let us apply $\mathrm{T}_{m-1, q-1}^{(h)}$ to (29). Since $\mathrm{T}_{m-1, q-1}^{(h)}\left(p_{r}(t ; h)\right)=0$, we obtain $\mathrm{T}_{m-1, q-1}^{(h)}\left(X_{a}(t ; h)\right)=0$ for $1 \leqslant a \leqslant 2(m-1)$, because $\mathrm{T}_{m-1, q-1}^{(h)}\left(R_{a}(t ; h)\right)=0$.

Therefore, for $2(m-1)+1 \leqslant r, a \leqslant q-1$ Eqs. (29) turn into a system of linear equations for the unknowns $\mathrm{T}_{m-1, q-1}^{(h)}\left(X_{a}(t ; h)\right)$. This system has only the zero solution, since its determinant $\left(t_{2 m-1} \ldots t_{q-m}\right)^{2(m-1)} V_{q-2 m+1}\left(t_{2 m-1}, \ldots, t_{q-m}\right)$ is nonzero. Since $\mathrm{T}_{m-1, q-1}^{(h)}\left(R_{2(m-1)+1}(t ; h)\right) \neq 0$, we obtain $\mathrm{T}_{m-1, q-1}^{(h)}\left(\varphi_{2(m-1)+1}(t ; h)\right)=\mathrm{T}_{m, q}^{(h)}(\widetilde{c}(t))=0$.

Corollary 5.3 (cf. [1]). A chain $c \in C_{q}\left(L^{(h)}(k)\right)$ is a stable cycle for $h=1$ if and only if $c(t)$ is divisible by $V_{q}^{3}(t)$ and for $h \neq 1$ if and only if $c(t)$ is divisible by $V_{q}\left(t^{3}\right)=V_{q}\left(t_{1}^{3} \delta t_{q}^{3}\right)$.

Proof. For $h=1$, Theorem 5.2 implies that $\widetilde{c}(t)$ is divisible by $t_{2}-t_{1}$ and hence, by symmetry, by $V_{q}^{2}(t)$. But then $c(t)$ is divisible by $V_{q}^{3}(t)$. For $h \neq 1$, Theorem 5.2 implies that $\widetilde{c}(t)$ is divisible by $\left(t_{2}-h t_{1}\right)\left(t_{2}-\bar{h} t_{1}\right)$. Then $c(t)$ is divisible by $\left(t_{2}-t_{1}\right)\left(t_{2}-h t_{1}\right)\left(t_{2}-\bar{h} t_{1}\right)=t_{2}^{3}-t_{1}^{3}$ and hence by $V_{q}\left(t^{3}\right)$. The sufficiency follows from (28).

Corollary 5.4. The polynomials $\left(\left(t_{1} \cdots t_{q}\right)^{k} V_{q}(t)\right)^{-1} \widehat{\varepsilon}_{[I ; J]}(t)$, where $[I ; J] \in \mathbf{N}(k),|I|+|J|=q$, and $|J|=m-1$, form a basis of the space $\operatorname{Sym}_{m, q}^{(h)}[t]$.

The dimension of the subspace of homogenous polynomials of degree $n$ is equal to the number of partitions $[I ; J] \in \mathbf{N}(1)$ with $|I|+|J|=q,|J|=m-1$, and $\|I\|=n+q(q+1) / 2$.

Proof. The first claim readily follows from Theorem 5.2 and Corollary 4.5. The second is the special case of the first for $k=1$.

\section{Stable Cycles and the Homology of the Algebras $L(k)$}

Theorem 6.1. The homology classes of the stable cycles $\widehat{\varepsilon}_{I}$, where $I \in \mathrm{M}_{q}(k)$, form a basis of the space $H_{q}\left(L^{(h)}(k)\right)$.

Proof. By Theorem 2.5, for $I_{0} \in \mathrm{M}_{q}(k)$ there exists a cocycle $\mathscr{C}_{I_{0}} \in C^{q}(k)$ such that $\left\langle\mathscr{C}_{I_{0}}, \widehat{\varepsilon}_{I}\right\rangle=$ $\delta_{I_{0}, I}$ for each $I \in \mathrm{M}_{q}(k)$. Since $\widehat{\varepsilon}_{I}$ is a cycle for each $I \in \mathbf{N}(k)$, it follows that the chain $z=$ $\sum_{I \in \mathrm{M}_{q}(k)} \beta_{I} \widehat{\varepsilon}_{I} \in C_{q}(k)$ represents a homology class in $H_{q}(k)$. If $z=d_{k}(x)$, then $\beta_{I_{0}}=\left\langle z, \mathscr{C}_{I_{0}}\right\rangle=$ $\left\langle d_{k}(x), \mathscr{C}_{I_{0}}\right\rangle=\left\langle x, \delta_{k}\left(\mathscr{C}_{I_{0}}\right)\right\rangle=0$. Thus, each nonzero vector in the space generated by the cycles $\widehat{\varepsilon}_{I}$, where $I \in \mathrm{M}_{q}(k)$, represents a nonzero $q$-dimensional class in $H_{q}(k)$. Since the vectors $\widehat{\varepsilon}_{I}$ are linearly independent, this completes the proof, because $\operatorname{dim} H_{q}(k)=\left|\mathrm{M}_{q}(k)\right|$ by Theorem 2.5.

Theorem 6.2. The homology classes of the stable cycles

$$
Z_{I}(t)= \begin{cases}S_{I-3 \rho(q)}(t) V_{q}^{3}(t) & \text { if } h=1 \\ S_{I-3 \rho(q)}(t) V_{q}\left(t^{3}\right) & \text { if } h \neq 1\end{cases}
$$

where $I \in \mathrm{M}_{q}(k)$, form a basis of the space $H_{q}\left(L^{(h)}(k)\right)$.

Proof. It suffices to prove the formula

$$
Z_{I}(t)=\widehat{\varepsilon}_{I}(t)+\sum_{I^{\prime} \triangleright I} \alpha_{I^{\prime}} \widehat{\varepsilon}_{I^{\prime}}(t) .
$$


Indeed, if $I \in \mathrm{M}(k)$ and $I^{\prime} \triangleright I$ is a nonsingular $k$-partition, then $I^{\prime} \in \mathrm{M}(k)$. Therefore, if $I \in \mathrm{M}(k)$, then all $I^{\prime}$ in formula (30) belong to $\mathrm{M}(k)$, and the matrix of transition from the set of chains $Z_{I}(t)$ to the set of chains $\widehat{\varepsilon}_{I}(t)$ is upper triangular in the linear order on $\mathbf{D}(k)$ consistent with $\sharp$. By Theorem 6.1 , the chains $\widehat{\varepsilon}_{I}$ form a basis of the homology space. Thus, the same is true for the set of chains $Z_{I}(t)$.

Note that $Z_{I}(t)=\Delta_{I}(t)+\sum_{I^{\prime} \triangleright I} \alpha_{I^{\prime}} \Delta_{I^{\prime}}(t)$. For $h \neq 1$, this follows from the expression (26) applied to the product of the polynomials $S_{I-3 \rho(q)}(t)$ and $S_{2 \rho(q)}(t)$ and multiplied by $V_{q}(t)$. For $h=1$, this follows from the expressions (26) and (27).

Thus, $Z_{I}(t)=\Delta_{I}(t)+\sum_{I^{\prime} \triangleright I} \alpha_{I^{\prime}} \Delta_{I^{\prime}}(t)+\sum_{I^{\prime \prime} \triangleright I} \alpha_{I^{\prime \prime}} \Delta_{I^{\prime \prime}}(t)$, where the $I^{\prime}$ are nonsingular $k$-partitions and the $I^{\prime \prime}$ are singular ones. The chain $c(t)=Z_{I}(t)-\widehat{\varepsilon}_{I}(t)-\sum_{I^{\prime} \triangleright I} \alpha_{I^{\prime}} \widehat{\varepsilon}_{I^{\prime}}(t)$ is a stable cycle by Proposition 5.3.

The definition of $\widehat{\varepsilon}_{I}$ implies that $\widehat{\varepsilon}_{I}(t)=e_{I}(t)+\sum_{\widetilde{I}} \beta_{\widetilde{I}} e_{\widetilde{I}}$, where the $k$-partitions $\widetilde{I}$ are singular. Therefore, $c$ is a linear combination of singular monomials. Since $c$ is a stable cycle, it follows by Theorem 4.3 that $\left\langle c, \mathrm{E}_{1}(k)\right\rangle=0$. On the other hand, $\left\langle c, e_{I}\right\rangle=0$ for each nonsingular $k$-partition $I$. By Theorem 2.4, the set of such cycles $e_{I}$, together with a basis of $\mathrm{E}_{1}(k)$, forms a basis of the space $C_{*}(k)$. Hence $c=0$. This completes the proof of formula (30).

\section{Laplace Operators of the Algebras $L^{(h)}(1)$}

Definition 7.1. The endomorphism $\Gamma_{k}^{(h)}=d_{k} \delta_{k}+\delta_{k} d_{k}$ of the space $C_{*}(k ; \mathbb{R})=C_{*}(k) \otimes \mathbb{R}$ is called the Laplace operator, and the elements of its kernel are called the harmonic chains of the algebra $L(k)$.

Obviously, $\Gamma_{k}^{(h)}$ is a self-adjoint operator commuting with $d_{k}$ and $\delta_{k}$. The complex $C_{*}(k ; \mathbb{R})$ is the direct sum of eigenspaces of $\Gamma_{k}^{(h)}$, which are subcomplexes of $C_{*}(k ; \mathbb{R})$. For nonzero eigenvalues, these complexes are acyclic, and the space of harmonic chains is isomorphic to $H_{*}(k ; \mathbb{R})$. This is a standard general claim (see [4]).

Definition 7.2 (B. L. Feigin). A linear endomorphism $A$ of the vector space $\Lambda(V)$ is called a second-order operator of degree $m$ if $A\left(\Lambda^{q}(V)\right) \subset \Lambda^{q+m}(V)$ and

$$
A\left(v_{1} \wedge \cdots \wedge v_{q}\right)=\sum_{1 \leqslant i<j \leqslant q}(-1)^{i+j-1} A_{i, j}\left(v_{1} \wedge \cdots \wedge v_{q}\right)-(q-2) \sum_{1 \leqslant i \leqslant q} A_{i}\left(v_{1} \wedge \cdots \wedge v_{q}\right)
$$

for arbitrary $v_{1}, \ldots, v_{q} \in V(q \geqslant 2)$, where $A_{i, j}\left(v_{1} \wedge \cdots \wedge v_{q}\right)=A\left(v_{i} \wedge v_{j}\right) \wedge v_{1} \wedge \cdots \wedge \widehat{v}_{i} \wedge \cdots \wedge \widehat{v}_{j} \wedge \cdots \wedge v_{q}$ and $A_{i}\left(v_{1} \wedge \cdots \wedge v_{q}\right)=v_{1} \wedge \cdots \wedge A\left(v_{i}\right) \wedge \cdots \wedge v_{q}$.

One can readily prove that

$$
A\left(u_{1} \wedge \cdots \wedge u_{q}\right)=\sum_{1 \leqslant i<j \leqslant q}(-1)^{\omega(i, j)} A_{i, j}\left(u_{1} \wedge \cdots \wedge u_{q}\right)-(q-2) \sum_{1 \leqslant i \leqslant q} A_{i}\left(u_{1} \wedge \cdots \wedge u_{q}\right)
$$

for $u_{1} \in \Lambda^{r_{1}}(V), \ldots, u_{q} \in \Lambda^{r_{q}}(V)$ and $q \geqslant 2$, where $\omega(i, j)=r_{i}\left(r_{1}+\cdots+r_{i-1}\right)+r_{j}\left(r_{1}+\cdots+\right.$ $\left.r_{j-1}\right)-r_{i} r_{j}$

Lemma 7.3. $\Gamma_{k}^{(h)}$ is a second-order operator of degree 0 .

Proof. The proof goes by a formal verification that uses only the fact that $d_{k}$ is a second-order operator and $\delta_{k}$ acts by formula (8).

Now let us study the Laplace operator $\Gamma^{(h)}=\Gamma_{1}^{(h)}$ of the algebra $L^{(h)}(1)$. For $I=\left[i_{1}, \ldots, i_{q}\right]$, let $\kappa_{h}(I)=\kappa_{h}\left(i_{1}\right)+\cdots+\kappa_{h}\left(i_{q}\right)$ and $(I)_{h}=\left(i_{1}\right)_{h}+\cdots+\left(i_{q}\right)_{h}$, where $\kappa_{h}(i)$ is defined by formula (2).

Theorem 7.4. Let $\lambda^{(h)}(I)=\kappa_{h}(I)-\left((I)_{h}^{2}-(I)_{h}\right) / 2$. Then

$$
\Gamma^{(h)}\left(\varepsilon_{[I ; J]}\right) \approx \lambda^{(h)}(I) \varepsilon_{[I ; J]} .
$$

Moreover, $\Gamma^{(h \neq 1)}\left(\varepsilon_{[I ; J]}\right)=\lambda^{(h)}(I) \varepsilon_{[I ; J]}$. In particular, the spectrum of $\Gamma^{(h)}$ coincides with the set of numbers $\lambda^{(h)}(I)$, where $I \in \mathrm{N}(1)$. 
Proof. One can readily verify that $\Gamma^{(h)}\left(e_{i}\right)=\lambda^{(h)}(i) e_{i}$. Therefore, $\Gamma^{(h)}\left(\delta_{1} e_{i}\right)=\lambda^{(h)}(i) \delta_{1} e_{i}$. This proves formula (32) for $\varepsilon(1)$-monomials of length 1 .

Let $D^{(h)}\left(e_{I}\right)=\Gamma^{(h)}\left(e_{I}\right)-\lambda^{(h)}(I) e_{I}$. A straightforward computation shows that

$$
\left\langle D^{(h)}\left(e_{i} \wedge e_{j}\right), e_{u} \wedge e_{v}\right\rangle=\left\{\begin{array}{ll}
(3 i)_{h}(v-u)_{h} & \text { if } i \leqslant u<v \leqslant j, \\
(3 u)_{h}(j-i)_{h} & \text { if } u<i<j<v,
\end{array} \quad i+j=u+v .\right.
$$

It follows that $\Gamma^{(h \neq 1)}\left(e_{i} \wedge e_{j}\right)=\lambda^{(h)}(i, j) e_{i} \wedge e_{j}$, because $(3 m)_{h \neq 1}=0$. Since $\Gamma^{(h)}$ is a secondorder operator, we obtain $\Gamma^{(h \neq 1)}\left(e_{I}\right)=\lambda^{(h)}(I) e_{I}$. Thus, $\Gamma^{(h \neq 1)}=p-\left(e_{0}^{2}-e_{0}\right) / 2$, where the linear mappings $e_{0}$ and $p$ are defined by the formulas $e_{0}\left(e_{I}\right)=(I)_{h} e_{I}$ and $p\left(e_{I}\right)=\kappa_{h}(I) e_{I}$. Keeping in mind that $e_{0}$ commutes with $\delta_{1}$ and that $p$ commutes with $\delta_{1}$ for $h \neq 1$, we obtain formula (32).

Let $h=1$. The directly verifiable formulas

$$
\begin{aligned}
\Gamma^{(1)}\left(e_{i} \wedge e_{j}\right) & =\lambda^{(1)}(i, j) e_{i} \wedge e_{j}-3(i+j) \sum_{1 \leqslant r<i} r e_{i-r} \wedge e_{j+r}+3 i \delta_{1} e_{i+j}, \\
\Gamma^{(1)}\left(\delta_{1} e_{i} \wedge e_{j}\right) & =\lambda^{(1)}(i, j) \delta_{1} e_{i} \wedge e_{j}-3 \sum_{1 \leqslant r<i} r\left((i+2 j+2 r) \delta_{1} e_{i-r} \wedge e_{j+r}+(i-2 r) e_{i-r} \wedge \delta_{1} e_{j+r}\right)
\end{aligned}
$$

prove (32) for the $\varepsilon(1)$-monomials $e_{i} \wedge e_{j}$ and $\delta_{1} e_{i} \wedge e_{j}$, since $j-i \geqslant 3$. They also imply the desired result for the $\varepsilon(1)$-monomials $e_{i} \wedge \delta_{1} e_{j}$ and $\delta_{1} e_{i} \wedge \delta_{1} e_{j}$, since

$$
\Gamma^{(1)}\left(e_{i} \wedge \delta_{1} e_{j}\right)=\Gamma^{(1)}\left(\delta_{1} e_{i} \wedge e_{j}\right)-\delta_{1} \Gamma^{(1)}\left(e_{i} \wedge e_{j}\right), \quad \Gamma^{(1)}\left(\delta_{1} e_{i} \wedge \delta_{1} e_{j}\right)=\delta_{1} \Gamma^{(1)}\left(\delta_{1} e_{i} \wedge e_{j}\right) .
$$

Thus, formula (32) is true for the $\varepsilon(1)$-monomials of length 2 for $h=1$.

Let $\varepsilon_{[I ; J]}=e_{\left(i_{1}\right)} \wedge \cdots \wedge e_{\left(i_{q}\right)}$ be an arbitrary $\varepsilon(1)$-monomial, where $q>2$. In formula (31), let $A=\Gamma^{(h)}$ and $u_{a}=e_{\left(i_{a}\right)}$. Using the expressions obtained for the $\varepsilon(1)$-monomials of lengths 1 and 2 and Lemma 2.3, we see that $\Gamma^{(1)}\left(\varepsilon_{[I ; J]}\right) \approx \lambda(I) \varepsilon_{[I ; J]}$, where

$$
\lambda(I)=\sum_{1 \leqslant a<b \leqslant q} \lambda^{(1)}\left(i_{a}, i_{b}\right)-(q-2) \sum_{1 \leqslant a \leqslant q} \lambda^{(1)}\left(i_{a}\right)=\lambda^{(1)}(I) .
$$

Formula (32) now follows by Theorem 2.4.

Corollary 7.5. $\operatorname{dim} H_{q}\left(L^{(h)}(1)\right)=2$ for $q>0$.

Proof. In view of the one-to-one correspondence between homology classes and harmonic chains, it suffices to show that $\lambda^{(h)}(I)=0$ for $I \in \mathbf{N}(1)$ only if $I=\xi(1, q)$ or $I=\xi(2, q)$.

For $h=1$, the claim follows from the easy-to-verify formula

$$
\lambda^{(1)}(I)=\frac{1}{6}\left(\|I\|\left(i_{1}-1\right)\left(i_{1}-2\right)+\sum_{1 \leqslant a<q}\|I\|_{a+1}\left(i_{a}+i_{a+1}\right)\left(i_{a+1}-i_{a}-3\right)\right),
$$

where $\|I\|_{m}=i_{m}+\cdots+i_{q}$. Let $h \neq 1$. Since $\left\lfloor\frac{i+1}{3}\right\rfloor=\frac{i-(i)_{h}}{3}$, it follows from Theorem 7.4 that $\lambda^{(h \neq 1)}(I)=0$ only if

$$
\|I\|=\left(3(I)_{h}^{2}-(I)_{h}\right) / 2 .
$$

Since $\|I\| \geqslant\left(3 q^{2}-q\right) / 2$ and $\left|(I)_{h}\right| \leqslant q$, we obtain $(I)_{h}= \pm q$. Thus, $I=\xi(1, q)$ or $I=\xi(2, q)$.

Corollary 7.6. The equation $\lambda^{(1)}(I)=\lambda>0$, where $I \in \mathbf{N}(1)$, has at most finitely many solutions. Let $I_{1}, \ldots, I_{r(\lambda)}$ be all of its solutions, and let $m(\lambda)$ be the multiplicity of the eigenvalue $\lambda$ of $\Gamma^{(1)}$. Then $m(\lambda)=2^{\operatorname{ind}_{1} I_{1}}+\cdots+2^{\operatorname{ind}_{1} I_{r(\lambda)}}$.

Proof. Since $I \in \mathbf{N}(1)$, we see that the vector

$$
v=\left(\left(i_{1}-1\right)\left(i_{1}-2\right), i_{2}-i_{1}-3, \ldots, i_{q}-i_{q-1}-3\right)=\left(v_{1}, \ldots, v_{q}\right)
$$

is nonzero, and its coordinates are nonnegative by (33). It follows from the same formula that $\lambda=\lambda^{(1)}(I)>\frac{1}{6} \sum_{a=1}^{q}\|I\|_{a} v_{a}$. For $\lambda>0$, this inequality has at most finitely many solutions on the set of triples $\{q, I, v\}$, where $I$ is a partition, $v \neq 0$ is an integer vector with nonnegative coordinates, and $|I|=\operatorname{dim}(v)=q$. This proves the first claim. 
If $\lambda^{(1)}(I)=\lambda$ and $V_{I}$ is the space spanned by the vectors $\varepsilon_{[I ; J]}$, then

$$
m(\lambda)=\operatorname{dim} \bigoplus_{I \in \mathbf{N}(1), \lambda^{(1)}(I)=\lambda>0} V_{I} .
$$

Since this sum is finite and $\operatorname{dim}\left(V_{I}\right)=2^{\operatorname{ind}_{1} I}$, we obtain the desired formula for $m(\lambda)$.

Corollary 7.7. One has $\Gamma^{(h)}\left(\operatorname{St}_{m}(1)\right) \subset \operatorname{St}_{m}(1)$. Moreover, $\Gamma^{(h \neq 1)}\left(\widehat{\varepsilon}_{[I ; J]}\right)=\lambda^{(h)}(I) \widehat{\varepsilon}_{[I ; J]}$ and

$$
\Gamma^{(1)}\left(\widehat{\varepsilon}_{[I ; J]}\right)=\lambda^{(1)}(I) \widehat{\varepsilon}_{[I ; J]}+\sum_{\left[I^{\prime} ; J^{\prime}\right] \operatorname{tr}[I ; J]} \alpha_{\left[I^{\prime} ; J^{\prime}\right]} \widehat{\varepsilon}_{\left[I^{\prime} ; J^{\prime}\right]} .
$$

In particular, the multiplicity of an eigenvalue $\lambda$ of $\Gamma^{(1)}$ on the space of stable cycles of $L_{1}$ is equal to the number of solutions of the equation $\lambda^{(1)}(I)=\lambda$, where $I \in \mathbf{N}(1)$.

Proof. Since $\Gamma^{(h)}\left(\mathrm{E}_{m}(1)\right) \subset \mathrm{E}_{m}(1)$ by Theorem 7.4, we obtain the first claim, because

$$
\left\langle\Gamma^{(h)}\left(\operatorname{St}_{m}(1)\right), \mathrm{E}_{m+1}(1)\right\rangle=\left\langle\operatorname{St}_{m}(1), \Gamma^{(h)}\left(\mathrm{E}_{m+1}(1)\right)\right\rangle=0 .
$$

For $h \neq 1$, the second claim is obvious, because $\Gamma^{(h \neq 1)}$ acts diagonally in the basis of $\varepsilon(1)$ monomials.

For $h=1$ and $\widehat{\varepsilon}_{[I ; J]} \in \operatorname{St}_{m}\left(L_{1}\right)$, the first claim implies the formula

$$
\Gamma^{(1)}\left(\widehat{\varepsilon}_{[I ; J]}\right)=\sum_{\left[I^{\prime} ; J^{\prime}\right],\left\|I^{\prime}\right\|=\|I\|,\left|J^{\prime}\right| \geqslant m} \alpha_{\left[I^{\prime} ; J^{\prime}\right]} \widehat{\varepsilon}_{\left[I^{\prime} ; J^{\prime}\right]}
$$

where $\alpha_{\left[I^{\prime} ; J^{\prime}\right]}=\left\langle\Gamma^{(1)}\left(\widehat{\varepsilon}_{[I ; J]}\right), \varepsilon_{\left[I^{\prime} ; J^{\prime}\right]}\right\rangle=\left\langle\widehat{\varepsilon}_{[I ; J]}, \Gamma^{(1)}\left(\varepsilon_{\left[I^{\prime} ; J^{\prime}\right]}\right)\right\rangle$.

Since $\Gamma^{(1)}\left(\varepsilon_{\left[I^{\prime} ; J^{\prime}\right]}\right)=\lambda^{(1)}\left(I^{\prime}\right) \varepsilon_{\left[I^{\prime} ; J^{\prime}\right]}+\ldots$ by formula $(32)$, we see that $\alpha_{[I ; J]}=\lambda^{(1)}(I)$ and $\alpha_{\left[I^{\prime} ; J^{\prime}\right]}=0$ if either $\left[I^{\prime} ; J^{\prime}\right] \triangleleft[I ; J]$ or $[I ; J]$ and $\left[I^{\prime} ; J^{\prime}\right]$ are incomparable.

Corollary 7.8 (cf. [1]). The stable cycle $Z_{I}(t)=S_{I-3 \rho(q)}(t) V_{q}^{3}(t) \in C_{*}\left(L_{1}\right)$, where $I \in \mathrm{N}(1)$ is a maximal partition with respect to $\sharp$, is an eigenvector of $\Gamma^{(1)}$. In particular, the harmonic chains of dimension $q>0$ of $L_{1}$ are exhausted by the chains $Z_{\xi(1, q)}(t)$ and $Z_{\xi(2, q)}(t)$.

Proof. The maximality of $I$ and formulas (30) imply that $Z_{I}(t)=\widehat{\varepsilon}_{I}$. By the same cause, formula (34) shows that $\widehat{\varepsilon}_{I}$ is an eigenvector of $\Gamma^{(1)}$.

Remark 7.9. For the algebras $L_{-1,0}^{(h)}$, the harmonic chains are exhausted by the chains $e_{-1} \wedge$ $e_{0} \wedge e_{1}$ and $e_{0}$, respectively. One can show that for $k \geqslant 1$

$$
\Gamma_{k}^{(h \neq 1)}=p+\frac{1}{2}\left((k+1)_{h}^{2} e_{0}-e_{0}^{2}-\sum_{r=1}^{k-1}\left(e_{r} e_{r}^{*}+e_{r}^{*} e_{r}\right)\right), \quad \text { where } p\left(e_{I}\right)=\frac{\|I\|-(I)_{h}}{3} e_{I}
$$

and $e_{r}^{*}$ is the operator dual to the adjoint action of $e_{r}$. This implies that

$$
\Gamma_{k}^{(h \neq 1)}= \begin{cases}p+\frac{1}{2}\left(e_{-1} e_{1}+e_{0}^{2}+e_{1} e_{-1}\right) & \text { if } k=-1, \\ p+\frac{1}{2}\left(e_{0}^{2}+e_{0}\right) & \text { if } k=0, \\ p-\frac{1}{2}\left(e_{0}^{2}-e_{0}\right) & \text { if } k=1, \\ p-\frac{1}{2}\left(e_{-1} e_{1}+e_{0}^{2}+e_{1} e_{-1}\right) & \text { if } k=2 .\end{cases}
$$

It is not difficult to obtain the spectral resolutions of $\Gamma_{k}^{(h \neq 1)}$ from these formulas for $k=-1,0,1,2$. One can show that the chains $e_{1}^{r}\left(e_{2} \wedge e_{5} \wedge \cdots \wedge e_{3 q-1}\right)$, where $r=0,1, \ldots, 2 q$, is a basis of the space of $q$-dimensional harmonic chains of $L_{2}^{(h \neq 1)}$ and that the stable filtration is invariant under the $\Gamma_{2}^{(h \neq 1)}$-action.

The spectra of $\Gamma_{k}^{(1)}$ for $k \neq 0,1$ and of $\Gamma_{k}^{(h \neq 1)}$ for $k>2$ contain complicated irrational values. But hopefully there exists a tame set of polynomials parameterized by $h$ and $k$ whose set of roots coincides with these spectra. The multiplicities of the eigenvalues of the operators $\Gamma_{k}^{(1)}$ are likely to be finite for all $k \geqslant-1$. 


\section{Addendum: A Combinatorial Proof of Theorem 3.6}

Let us construct a bijective map $S: \mathrm{D}_{q}^{(n)} \rightarrow \mathbf{N}_{q}^{(n)}$. Recall that the diagram of a partition $I=\left[i_{1}, \ldots, i_{q}\right]$ is the set of points $(a, b) \in \mathbb{Z} \times \mathbb{Z}$ (vertices) such that $1 \leqslant b \leqslant q$ and $1 \leqslant a \leqslant i_{b}$. The diagonal of the partition $I$ is the set of vertices with $a+b=q+1$.

Assume that the diagonal of $I$ contains $r$ vertices. Let us number them from bottom to top. Let $x_{i}$ be the number of diagram vertices located in the row to the right of the $i$ th diagonal vertex, including this vertex, and let $y_{i}$ be the number of diagram vertices located in the column strictly below the $i$ th vertex. We can interpret $I$ as a pair of integer sequences

$$
I=\left(x_{1}, \ldots, x_{r} \mid y_{1}, \ldots, y_{r}\right), \quad \text { where } 1 \leqslant x_{1}<\cdots<x_{r}, 0 \leqslant y_{1}<\cdots<y_{r} .
$$

Such a pair corresponds to a strict partition if and only if

(1) $x_{i+1}-x_{i} \geqslant 2$ for $i=1, \ldots, r-1$.

(2) $y_{i+1}-y_{i}=1$ or 2 for $i=1, \ldots, r-1$.

(3) $y_{1}=0$ or 1 .

(4) If $x_{1}=1$, then $y_{1}=0$.

Let $I=\left(x_{1}, \ldots, x_{r} \mid y_{1}, \ldots, y_{r}\right) \in \mathrm{D}_{q}^{(n)}$, and let $1 \leqslant a_{1}<\cdots<a_{s} \leqslant r$ be all the numbers such that $y_{a_{k}}-y_{a_{k}-1}=2$. (By definition, $y_{0}=-1$.) Set $S(I)=[\widetilde{I} ; \widetilde{J}]$, where

$$
\widetilde{I}=\left[x_{1}+y_{1}, \ldots, x_{r}+y_{r}\right], \quad \widetilde{J}=\left[x_{a_{1}}+y_{a_{1}}, \ldots, x_{a_{s}}+y_{a_{s}}\right] .
$$

Since $s=q-r$, it follows from conditions (1)-(4) that $S(I) \in \mathscr{N}_{q}^{(n)}$.

Now let us define a mapping $S^{-1}: \mathbf{N}_{q}^{(n)} \rightarrow \mathrm{D}_{q}^{(n)}$. Let $[I ; J] \in \mathbf{N}_{q}^{(n)}$, where $I=\left[i_{1}, \ldots, i_{|I|}\right]$. Set $S^{-1}[I ; J]=\left(x_{1}, \ldots, x_{|I|} \mid y_{1}, \ldots, y_{|I|}\right)$, where

$$
y_{1}=\left\{\begin{array}{ll}
0 & \text { if } i_{1} \notin J, \\
1 & \text { if } i_{1} \in J,
\end{array} \quad y_{a}=\left\{\begin{array}{ll}
y_{a-1}+1 & \text { if } i_{a} \notin J, \\
y_{a-1}+2 & \text { if } i_{a} \in J,
\end{array} \quad 2 \leqslant a \leqslant|I|,\right.\right.
$$

and $x_{a}=i_{a}-y_{a}$. Obviously, $\left|S^{-1}[I ; J]\right|=y_{|I|}+1=|I|+|J|=q$. Therefore, $S^{-1}[I ; J] \in \mathrm{D}_{q}^{(n)}$. The definitions imply that the mappings $S$ and $S^{-1}$ are mutually inverse. The proof of Theorem 3.6 is complete.

Example: for the partition $I=[2,3,5,6,8,9]=(2,4,7,9 \mid 0,2,4,5)$ with diagram

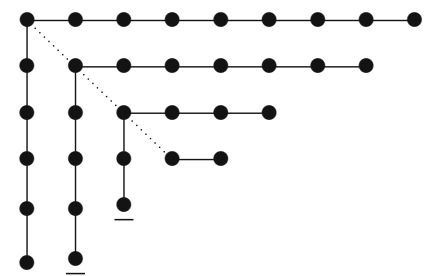

the parts of $S(I)$ are the numbers of vertices connected by the solid lines. Such a part is marked if the lowest corresponding vertex of the diagram is located strictly below the diagonal and there are no more vertices in the row to the right of this vertex. Thus, $S[2,3,5,6,8,9]=[2, \underline{6}, \underline{11}, 14]$.

Remark 8.1. Using Lemma 3.10 and the obtained combinatorial interpretation of Sylvester's identity, one can prove the following generalization:

$$
\prod_{i=k}^{\infty}\left(1+t x^{i}\right)=1+t x^{k} \frac{1+t x^{k+1}}{1-x}+\sum_{l=2}^{\infty} t^{l} x^{k l+3 l(l-1) / 2} \frac{\left(1+t x^{k}\right) \cdots\left(1+t x^{l+k-2}\right)\left(1+t x^{2 l+k-1}\right)}{(1-x) \cdots\left(1-x^{l-1}\right)\left(1-x^{l}\right)} .
$$

\section{References}

[1] B. L. Fer̆gin and D. B. Fuchs, "Homology of the Lie algebra of vector fields on the line," Funkts. Anal. Prilozhen., 14:3 (1980), 45-60; English transl.: Functional Anal. Appl., 14:3 (1980), 201-212. 
[2] B. L. Feigin and D. B. Fuchs, "Verma modules over the Virasoro algebra," in: Topology (Leningrad, 1982), Lecture Notes in Math., vol. 1060, Springer-Verlag, 1984, 230-245.

[3] B. L. Feigin, D. B. Fuchs, and V. S. Retakh, "Massey operations in the cohomology of the infinite-dimensional Lie algebra $L_{1}$," in: Topology and geometry-Rohlin Seminar, Lecture Notes in Math., vol. 1346, Springer-Verlag, 1988, 13-31.

[4] D. B. Fuchs, Cohomology of Infinite-Dimensional Lie Algebras, Contemp. Soviet Math., Consultants Bureau, New York, 1986.

[5] I. M. Gelfand, "The cohomology of infinite dimensional Lie algebras: some questions of integral geometry," in: Actes du Congrès International des Mathématiciens (Nice, 1970), Tome 1, Gauthier-Villars, Paris, 1971, 95-111.

[6] I. M. Gelfand, B. L. Fer̆gin, and D. B. Fuchs, "Cohomology of infinite-dimensional Lie algebras and Laplace operators," Funkts. Anal. Prilozhen., 12:4 (1978), 1-5; English transl.: Functional Anal. Appl., 12:4 (1978), 243-247.

[7] L. V. Gončarova, "Cohomology of Lie algebras of formal vector fields on the line," Funkts. Anal. Prilozhen., 7:2 (1973), 6-14; 7:3 (1973), 33-44; English transl.: Functional Anal. Appl., 7:2 (1973), 91-97; 7:3 (1973), 194-203.

[8] V. G. Kac, Infinite-Dimensional Lie Algebras, Cambridge University Press, Cambridge, 1990.

[9] Sh. Kumar, Kac-Moody groups, their flag varieties and representation theory, Progress in Math., vol. 204, Birkhäuser Boston Inc., Boston, MA, 2002.

[10] I. G. Macdonald, Symmetric Functions and Hall Polynomials, 2nd ed., With contributions by A. Zelevinsky, Oxford Math. Monographs, Oxford Science Publ., The Clarendon Press, Oxford Univ. Press, New York, 1995.

[11] A. Rocha-Caridi and N. R. Wallach, "Characters of irreducible representations of the Lie algebra of vector fields on the circle," Invent. Math., 72:1 (1983), 57-75.

[12] J. J. Sylvester and F. Franklin, "A constructive theory of partitions, arranged in three acts, an interact and an exodion," Amer. J. Math., 5:1-4 (1882), 251-330.

[13] F. V. Vaĭnshteŭn [F. V. Weinstein], "Filtering bases, cohomology of infinite-dimensional Lie algebras, and Laplace operators," Funkts. Anal. Prilozhen., 19:4 (1985), 11-22; English transl.: Functional Anal. Appl., 19:4 (1985), 259-269.

[14] F. V. Weinstein, "Filtering bases: a tool to compute cohomologies of abstract subalgebras of the Witt algebra," in: Unconventional Lie algebras, Adv. Soviet Math., vol. 17, Amer. Math. Soc., Providence, RI, 1993, 155-216.

Universität Bern, Institut Für AnAtomie

e-mail: Weinstein@ana.unibe.ch

Translated by F. V. Weinstein 